\title{
Reconnection studies under different types of turbulence driving
}

\author{
G. Kowal ${ }^{1}$, A. Lazarian ${ }^{2}$, E. T. Vishniac ${ }^{3}$, and K. Otmianowska-Mazur ${ }^{4}$ \\ ${ }^{1}$ Instituto de Astronomia, Geofísica e Ciências Atmosféricas, Universidade de São Paulo, Rua do Matão, 1226 - Cidade \\ Universitária, CEP 05508-090, São Paulo/SP, Brazil \\ ${ }^{2}$ Department of Astronomy, University of Wisconsin, 475 North Charter Street, Madison, WI 53706, USA \\ ${ }^{3}$ Department of Physics and Astronomy, McMaster University, 1280 Main Street West, Hamilton, ON L8S 4M1, Canada \\ ${ }^{4}$ Obserwatorium Astronomiczne, Uniwersytet Jagielloński, ul. Orla 171, 30-244 Kraków, Poland
}

Correspondence to: G. Kowal (kowal@astro.iag.usp.br)

Received: 28 November 2011 - Revised: 11 March 2012 - Accepted: 13 March 2012 - Published: 18 April 2012

\begin{abstract}
We study a model of fast magnetic reconnection in the presence of weak turbulence proposed by Lazarian and Vishniac (1999) using three-dimensional direct numerical simulations. The model has been already successfully tested in Kowal et al. (2009) confirming the dependencies of the reconnection speed $V_{\text {rec }}$ on the turbulence injection power $P_{\text {inj }}$ and the injection scale $l_{\text {inj }}$ expressed by a constraint $V_{\text {rec }} \sim P_{\text {inj }}^{1 / 2} l_{\text {inj }}^{3 / 4}$ and no observed dependency on Ohmic resistivity. In Kowal et al. (2009), in order to drive turbulence, we injected velocity fluctuations in Fourier space with frequencies concentrated around $k_{\mathrm{inj}}=1 / l_{\mathrm{inj}}$, as described in Alvelius (1999). In this paper, we extend our previous studies by comparing fast magnetic reconnection under different mechanisms of turbulence injection by introducing a new way of turbulence driving. The new method injects velocity or magnetic eddies with a specified amplitude and scale in random locations directly in real space. We provide exact relations between the eddy parameters and turbulent power and injection scale. We performed simulations with new forcing in order to study turbulent power and injection scale dependencies. The results show no discrepancy between models with two different methods of turbulence driving exposing the same scalings in both cases. This is in agreement with the Lazarian and Vishniac (1999) predictions. In addition, we performed a series of models with varying viscosity $v$. Although Lazarian and Vishniac (1999) do not provide any prediction for this dependence, we report a weak relation between the reconnection speed with viscosity, $V_{\mathrm{rec}} \sim v^{-1 / 4}$.
\end{abstract}

\section{Introduction}

Magnetic fields are observed in many astrophysical objects and usually play an important or even crucial role in their dynamics (see, e.g. Crutcher, 1999; Beck, 2002; Vallée, 1997, 1998). They are a key ingredient of astrophysical processes such as magneto-rotational instability, magnetic dynamo, transport and acceleration of cosmic rays, accretion disks, turbulence, solar phenomena, gamma ray bursts, etc. (Balbus and Hawley, 1998; Parker, 1992; Hanasz et al., 2009; KulpaDybeł et al., 2011; Schlickeiser and Lerche, 1985; Melrose, 2009; Elmegreen and Scalo, 2004; Kotera and Olinto, 2011).

Magnetic fields are solenoidal and evolve only through changes in the curl of the electric field. In the limit of zero resistivity the topology of the field lines is a constant of motion and the magnetic flux threading any fluid element is constant. Generating large scale magnetic fields requires some kind of battery effect, like the Biermann battery (Khanna, 1998) and generating strong large scale magnetic fields requires a dynamo (see Parker, 1992, for example). In the limit of very small resistivity, which is typical for astrophysical objects, the magnetic flux is "frozen in" and magnetic field lines will resist passing through one another or changing their topology (Moffat, 1978). Due to the presence of plasma motions, in particular turbulence, this would result in a very complex tangle of field lines in real objects, with negligible large scale magnetic flux. However, observations indicate that the mean and turbulent components of magnetic fields in many astrophysical objects are of similar strengths (see Beck, 2002, for example). This implies the existence of a process which can violate the frozen-in condition on dynamical time scales, i.e., fast magnetic field reconnection.

The first analytic model for magnetic reconnection was proposed independently by Parker (1957) and Sweet (1958). Sweet-Parker reconnection has the virtue that it relies on a robust and straightforward geometry. Two regions with uniform magnetic fields are separated by thin current sheet. The speed of reconnection is given roughly by the resistivity divided by the sheet thickness. However, the plasma in the current sheet is constrained to move along the local field lines and is ejected from the edge of the current sheet at the Alfvén speed, $V_{\mathrm{A}}$. Since the width of the current sheet limits the flux 
of expelled plasma, the overall reconnection speed is reduced from the Alfvén speed by the square root of the Lundquist number, $S \equiv L V_{\mathrm{A}} / \eta$, where $\eta$ is the resistivity and $L$ is the length of the current sheet. In most astrophysical contexts $S$ is very large and the Sweet-Parker reconnection speed, $V_{\mathrm{SP}} \approx V_{\mathrm{A}} S^{-1 / 2}$, is negligible. Fast reconnection requires that the dependence on $\eta$ be erased. Given the simplicity of the Sweet-Parker setup, this requires that the simple geometry of the current sheet must be broken.

The realisation that Sweet-Parker reconnection is inadequate to explain magnetic reconnection in an astrophysical context was immediately apparent and gave rise to decades of research on models of fast reconnection (see Biskamp, 2000; Priest and Forbes, 2000, for reviews). The first proposal was to replace the current sheet with an X-point configuration, so that the "sheet" thickness and length are comparable. This is the basis for Petschek's model of fast reconnection (Petschek, 1964). However, a dynamically self-consistent Xpoint requires that the outflow prevent a general collapse into a narrow current sheet. Otherwise we would expect that the same bulk forces that brought the magnetic field lines together would lead to Sweet-Parker reconnection. Petschek (1964) proposed that slow-mode shocks on either side of the $\mathrm{X}$-point would serve this purpose. Moreover, those shocks are responsible for converting most of the magnetic energy into the heat and kinetic energy. The X-point in this model has an overall size which depends on resistivity, but since the magnetic field decrease logarithmically when approaching the current sheet (due to the assumption of the currentfree magnetic field in the inflow region), the resulting reconnection speed is some fraction of $V_{\mathrm{A}}$. Numerical simulations with uniform resistivity (Biskamp, 1996) have showed that in the MHD limit the shocks fade away and the contact region expands into a sheet. The only way to make the Petschek configuration stable is by introducing the local non-uniform resistivity (Ugai and Tsuda, 1977; Scholer, 1989; Ugai, 1992; Yan et al., 1992; Forbes, 2000; Shibata and Magara, 2011).

This leaves the possibility that $\mathrm{X}$-point reconnection is stable when the plasma is collisionless. Numerical simulations (Shay et al., 1998, 2004) have been encouraging. However, there are several important issues that remain unresolved. First, it is not clear that this kind of fast reconnection persists on scales greater than the ion inertial scale (see Bhattacharjee et al., 2003). Several numerical studies (Wang et al., 2001; Smith et al., 2004; Fitzpatrick, 2004) have found large scale reconnection speeds which depend on resistivity, i.e., are not fast. Second, in many circumstances the magnetic field geometry does not allow the formation of X-point reconnection. For example, a saddle-shaped current sheet cannot be spontaneously replaced by an X-point. The energy required to do so is comparable to the magnetic energy liberated by reconnection and must be available beforehand. Finally, the requirement for reconnection occurrance in a collisionless plasma restricts this model to a small fraction of astrophysical applications. For example, while reconnec- tion in stellar coronae might be described in this way, stellar chromospheres are not. More generally Yamada (2007) estimated that the scale of the reconnection sheet should not exceed about 40 times the electron mean-free path. This condition is not satisfied in many environments which one might naively consider to be collisionless, among them the interstellar medium. The conclusion that stellar interiors and atmospheres, accretion disks, and the interstellar medium in general does not allow fast reconnection is drastic and unpalatable.

An alternative to the X-point geometry is to consider magnetic fields that are chaotic, even if only weakly so. Requiring the plasma to flow along the local magnetic field implies a powerful constraint on reconnection, only if the field lines themselves are laminar. Lazarian and Vishniac (1999, hereinafter LV99) proposed a model for fast reconnection which depends on the presence of turbulence and its production of weakly stochastic field lines (also briefly described in Sect. 2). Turbulence is a natural consequence of convection in stars and of the magnetorotational instability in accretion disks (for a review see Balbus and Hawley, 1998). In addition, it is now generally accepted that the "Big Power Law in the Sky" indicates the presence of turbulence on scales from tens of parsecs to thousands of kilometres (Armstrong et al., 1995; Chepurnov and Lazarian, 2010). Among other sources, evidence for this comes from studies of atomic hydrogen spectra in molecular clouds and galaxies (see Lazarian and Pogosyan, 2000; Stanimirović and Lazarian, 2001; Padoan et al., 2006, 2009; Chepurnov et al., 2010, see also review by Lazarian, 2009 and references therein), as well as recent studies of emission lines and Faraday rotation (see Burkhart et al., 2010; Gaensler et al., 2011). LV99's model uses the properties of turbulence to predict broad outflows from extended current sheets. The diffusivity of magnetic field line trajectories in a turbulent plasma implies that flows can follow local magnetic field lines without being confined to the current sheet. When the turbulent diffusivity is less than the ohmic resistivity, this model reduces to the SweetParker reconnection model.

The first test of the LV99 model using three-dimensional (3-D) MHD simulations was performed in Kowal et al. (2009). The main predictions of the model were confirmed. In this paper, we provide additional numerical evidence of magnetic reconnection in turbulent environments by testing different mechanisms for injecting turbulence. In Sect. 2, we briefly review the LV99 model of reconnection and its theoretical predictions. In Sect. 3, we describe, in detail, the numerical model studied in this paper and the new method of turbulence driving. Although the initial setup and boundary conditions are similar to our previous studies and, described in detail in Kowal et al. (2009), we briefly describe them here for completeness, as well. In Sect. 4, we present an extensive description of new results obtained from studying our numeric model, which we discuss later in Sect. 5. In Sect. 6, we present our main conclusions. 


\section{The Lazarian-Vishniac (1999) model}

The notion that turbulence can influence reconnection rate is not unprecedented. The ideas in this regard were discussed long before LV99. However, they fell short of solving the problem. For instance, Speiser (1970) considered the effects of turbulence on microscopic resistivity, Jacobson and Moses (1984) proposed that the current diffusivity should be modified to include the diffusion of electrons across the large scale magnetic field due to the small scale field line stochasticity. The consequent modifications to the ohmic resistivity have only a marginal effect on the Sweet-Parker reconnection speeds. Matthaeus and Lamkin $(1985,1986)$ studied 2-D magnetic reconnection in the presence of external turbulence both theoretically and numerically. They pointed out various turbulence mechanisms that would enhance reconnection rates, including multiple $\mathrm{X}$-points as reconnection sites. However, this work did not include the effect of magnetic field wandering, which is at the core of the LV99. They did not provide analytical predictions of the reconnection speed either ${ }^{1}$.

We begin by offering a brief summary of the differences between the Sweet-Parker model of the laminar reconnection (Parker, 1957; Sweet, 1958) and the Lazarian-Vishniac model which accounts for the effects of turbulence (Lazarian and Vishniac, 1999). The latter can be seen as a generalization of the Sweet-Parker model (see Fig. 1) in the sense that the two regions of differing magnetic directions are pressed up against one another over a broad contact region. This is a generic configuration, which should arise naturally whenever a magnetic field has a non-trivial configuration, whose energy could be lowered through reconnection. The outflow of plasma and reconnected flux will fluctuate as the turbulence evolves and the field line connections change, but the long-term average will reflect the turbulent diffusion of the field lines. Consequently, the essential difference between the Sweet-Parker model and the LV99 model is that the former outflow is limited by microphysical Ohmic diffusivity, while in the LV99 model the large-scale magnetic field wandering determines the thickness of outflow. For extremely weak turbulence, when the range of magnetic field wandering becomes smaller than the width of the Sweet-Parker layer $L S^{-1 / 2}$, the two models are indistinguishable. By weak turbulence, following LV99, we understand a regime where the correlation length is much greater than the distance by which individual field lines deviate from a straight line.

LV99 considered a large scale, well-ordered magnetic field, of the kind that is normally used as a starting point for discussions of reconnection. In the presence of turbu-

\footnotetext{
${ }^{1}$ At the same time, after LV99 was published, Kim and Diamond (2001) produced a study arguing that turbulence will not change reconnection rates in the Sweet-Parker geometry. This study has been criticized by Lazarian et al. (2004) and Eyink et al. (2011). The present paper provides numerical evidence that the reconnection rates do increase in the presence of turbulence.
}
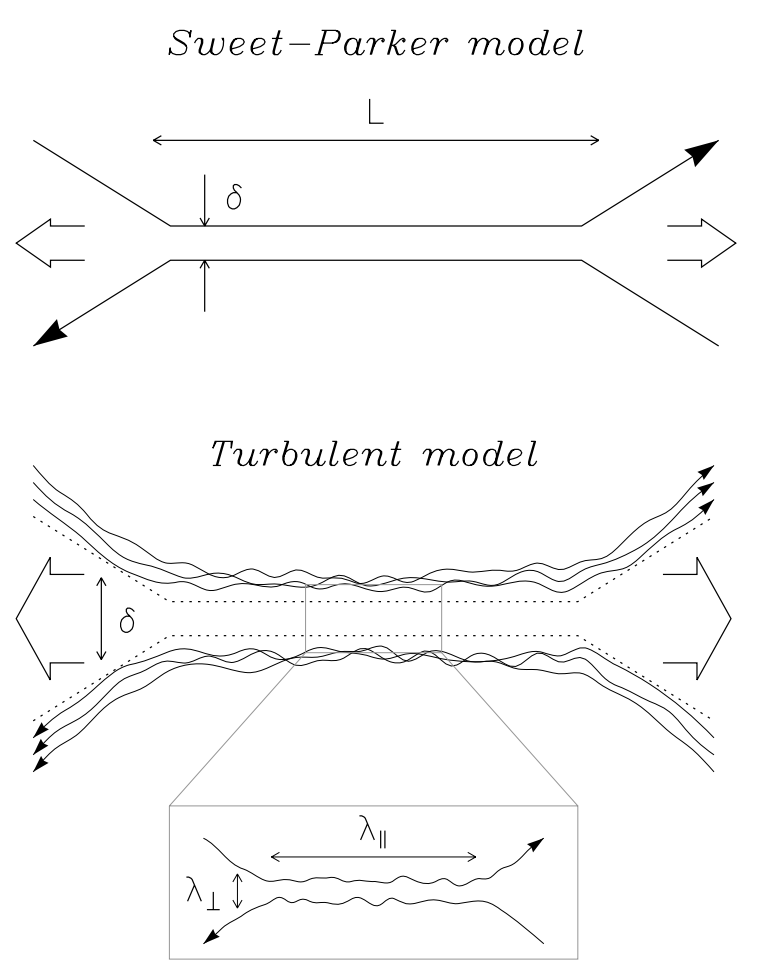

Fig. 1. Upper plot: The Sweet-Parker reconnection model. The outflow is confined to a thin layer of $\delta$, which is set by Ohmic diffusivity. The length of the current sheet is a macroscopic scale $L \gg \delta$. Magnetic field lines are assumed to be laminar. Middle plot: Reconnection in the presence of stochastic magnetic field lines. The stochasticity introduced by turbulence is weak and the mean field is clear direction. The outflow width is set by the diffusion of the magnetic field lines, which is a macroscopic process, independent of resistivity. Low plot: An individual small scale reconnection region. The reconnection over small patches of magnetic field determines the local reconnection rate. The global reconnection rate is substantially larger as many independent patches reconnect simultaneously. Conservatively, the LV99 model assumes that the small scale events happen at a slow Sweet-Parker rate. Following Lazarian et al. (2004) and Kowal et al. (2009).

lence, the field has some small scale 'wandering'. LV99 suggested that the presence of a random magnetic field component leads to fast reconnection. There are three phenomena mainly responsible for this:

- only a small fraction of any magnetic field line is subject to direct Ohmic annihilation, therefore, the fraction of magnetic energy that goes directly into heating the fluid drops down to zero as the fluid resistivity vanishes,

- the presence of turbulence enables many magnetic field lines to enter the reconnection zone simultaneously,

- turbulence broadens the width of the ejection thickness allowing for more efficient removal of the reconnected flux. 
With the Goldreich and Sridhar (1995, henceforth GS95) model of turbulence, LV99 obtained:

$V_{\mathrm{rec}}=V_{\mathrm{A}}\left(\frac{l}{L}\right)^{1 / 2}\left(\frac{v_{l}}{V_{\mathrm{A}}}\right)^{2}$,

where $l$ and $v_{l}$ are the energy injection scale and velocity. This expression assumes that energy is injected isotropically at the scale $l$ smaller than the length of current sheet $L$, which for sub-Alfvénic turbulence leads to the generation of weakly interacting waves at that scale. The waves transfer energy to modes with larger values of $k_{\perp}$ until strong turbulence sets in. It is important to note that the strongly turbulent eddies have a characteristic velocity of $v_{\mathrm{turb}} \approx V_{\mathrm{A}}\left(v_{l} / V_{\mathrm{A}}\right)^{2}$. In other words, the reconnection speed is the large eddy strong turbulent velocity times factors which depend on whether the current sheet is smaller or larger than the large eddies (whose length is approximately the injection scale). In this sense, the reconnection speed should be fairly insensitive to the exact mechanism for turbulent power injection. The main purpose of this paper is test whether or not this is true for a simple modification of the driving mechanism used in Kowal et al. (2009).

It is important to note three features of Eq. (1). First, and most important, it is independent of resistivity. This is, by definition, fast reconnection. Second, we usually expect reconnection to be close to the turbulent eddy speed, the geometric ratios that enter the expression, i.e., the injection scale $l$ divided by the length of the reconnection layer $L$, are typically of the order of unity. Reconnection will occur on dynamical time scales. Finally, we note that, in particular situations when turbulence is extremely weak, the reconnection speed can be much slower than the Alfvén speed.

More recently, Eq. (1) was derived using the ideas based on the well-known concept of Richardson diffusion (Eyink et al., 2011). From the theoretical perspective this new derivation avoids rather complex considerations of the cascade of reconnection events that were presented in LV99 to justify the model. Eyink et al. (2011) also shows that LV99 model is closely connected to the recently developed idea of "spontaneous stochasticity" of magnetic fields in turbulent fluids.

In general, the situation in the reconnection community now is very different from that of a decade ago. Currently, possibilities of fast reconnection in MHD regime due to instabilities of the reconnection layers are widely discussed (Loureiro et al., 2009; Bhattacharjee et al., 2009). These ideas can be traced back to the work of Shibata and Tanuma (2001). The instabilities, like tearing instability, open up the reconnection layer enabling a wide outflow. We expect such an outflow to become turbulent for most of astrophysical conditions. In this case, the process can be important for initiating reconnection in the particular situation when the level of pre-existing turbulence is initially low to initiate sufficiently fast reconnection. We feel that exploring the ways of initiation of turbulent reconnection is very synergistic to the LV99 ideas, but in the current paper, we focus on the case of preexisting turbulence of sufficient level. This is the primary domain for which LV99 provides predictions.

Given the limited dynamical range of numerical simulations, we can only inject power on scales less than $L$. The most convenient numerical parameter is not $v_{l}$, but the energy injection power $P$. The power in the turbulent cascade is $P \sim v_{\text {turb }}^{2}\left(V_{\mathrm{A}} / l\right)$ or $v_{l}^{4} /\left(l V_{\mathrm{A}}\right)$. The amount of energy injected during one Alfvén time unit $t_{\mathrm{A}} \equiv L / V_{\mathrm{A}}$, which is constant in our models, is $t_{\mathrm{A}} P \sim\left(L / V_{\mathrm{A}}\right) v_{l}^{4} /\left(l V_{\mathrm{A}}\right)$. Therefore, $v_{l}^{2} \sim(l / L)^{1 / 2}\left(P t_{\mathrm{A}}\right)^{1 / 2} V_{\mathrm{A}}$. Substituting $v_{l}^{2}$ in Eq. (1) results in

$V_{\text {rec }} \sim\left(\frac{l}{L}\right)\left(t_{\mathrm{A}} P\right)^{1 / 2} \propto l P^{1 / 2}$,

which is the prediction we will test here. In what follows, we refer to the injection power and scale using $P_{\text {inj }}$ and $l_{\text {inj }}$, respectively.

\section{Numerical setup}

\subsection{Governing equations}

We use a high-order shock-capturing Godunov-type scheme based on the monotonicity preserving (MP) spatial reconstruction (see, e.g. Suresh and Huynh, 1997; He et al., 2011) and Strong Stability Preserving Runge-Kutta (SSPRK) time integration (see Gottlieb et al., 2009, and references therein) to solve isothermal non-ideal MHD equations,

$$
\begin{aligned}
& \frac{\partial \rho}{\partial t}+\nabla \cdot(\rho \boldsymbol{v})=0, \\
& \frac{\partial \rho \boldsymbol{v}}{\partial t}+\nabla \cdot\left[\rho \boldsymbol{v} \boldsymbol{v}+\left(a^{2} \rho+\frac{B^{2}}{8 \pi}\right) I-\frac{1}{4 \pi} \boldsymbol{B} \boldsymbol{B}\right]=\boldsymbol{f}, \\
& \frac{\partial \boldsymbol{A}}{\partial t}+\boldsymbol{E}=\boldsymbol{g},
\end{aligned}
$$

where $\rho$ and $\boldsymbol{v}$ are plasma density and velocity, respectively, $\boldsymbol{A}$ is the vector potential, $\boldsymbol{E}=-\boldsymbol{v} \times \boldsymbol{B}+\eta \boldsymbol{J}$ is the electric field, $\boldsymbol{B} \equiv \nabla \times \boldsymbol{A}$ is the magnetic field, $\boldsymbol{J}=\nabla \times \boldsymbol{B}$ is the current density, $a$ is the isothermal speed of sound, $\eta$ is the resistivity coefficient, and $\boldsymbol{f}$ and $\boldsymbol{g}$ represent the turbulence driving terms either in velocity or vector potential. We used a multi-state Harten-Lax-van Leer (HLLD, Mignone, 2007) approximate Riemann solver for solving the isothermal MHD equations. The HLLD Riemann solver takes into account magnetic fields and can follow Alfvén waves with minimal numerical dissipation. This is particularly important here, because our simulations are in the quasi-incompressible regime, where most of energy is transported by Alfvén waves. The $\nabla \cdot \boldsymbol{B}=0$ is maintained by solving the induction equation (Eq. 5) using the field interpolated constrained transport (CT) scheme based on a staggered mesh (e.g. Londrillo and Del Zanna, 2000; Tóth, 2000). 


\subsection{Model description and initial conditions}

Our reconnection simulation setup is illustrated in Fig. 2, which is a 2-D cut through the problem setup, indicating the location of the diffusion region. The top and bottom of the computational domain contain equal and opposite field components in the $\hat{x}$ direction, as well as a shared component $B_{z}$ (see the left panel of Fig. 2). Magnetic field lines enter through the top and bottom and are bent by the inflow $V_{\text {in }}$ as they move into the diffusion region. The diffusion region has a length $\Delta$ in the $\hat{x}$ direction and a thickness $\delta$ in the $\hat{y}$ direction (see the left panel of Fig. 2). The box is periodic in the $\hat{z}$ direction and the diffusion region extends through the entire domain. The projection of the magnetic topology on the $\mathrm{XZ}$ plane shows that the lines in the upper region (solid lines in the right panel of Fig. 2) and in the lower region (dashed lines) create an angle $\alpha$ determined by the strength of the shared component $B_{0 z}$. Once the incoming magnetic lines enter the diffusion region, they are reconnected and the product of this process is ejected along $\mathrm{X}$ direction with a speed $V_{\text {out }}$ (the left panel of Fig. 2).

We begin with a Harris current sheet of the form $B_{x}(x, y, z)=B_{0 x} \tanh (y / \theta)$ initialised using the magnetic vector potential $A_{z}(x, y, z)=\ln |\cosh (y / \theta)|$, and a uniform guide field $B_{z}(x, y, z)=B_{0 z}=$ const. The initial setup is completed by setting the density profile from the condition of the uniform total (thermal plus magnetic) pressure $p_{\text {tot }}(t=0, x, y, z)=$ const and setting the initial velocity to zero.

Magnetic reconnection is initiated by a small perturbation of the vector potential $\delta A_{z}(x, y, z)=\delta B_{0 x} \cos (2 \pi x)$ to the initial configuration of $A_{z}(t=0, x, y, z)$ whose strength is given by the coefficient $\delta B_{0 x}$.

In all our simulations, the strength of the magnetic field is expressed in terms of the Alfvén velocity defined by the anti-parallel component of the unperturbed magnetic field. Similarly the density is expressed in terms of the unperturbed density $\rho_{0}=1$ and velocities are expressed as fractions of the fiducial Alfvén speed. The length of the box in the $\hat{x}$ direction defines the unit of distance and time is measured in units of $L_{x} / V_{\mathrm{A}}$. In the new set of models, we set the initial strength of the anti-parallel magnetic field component $B_{0 x}=1.0$ and the guide field $B_{0 z}=0.1$. We performed modelling for two resistivity coefficients $\eta_{u}=5 \cdot 10^{-4}$ and $\eta_{u}=10^{-3}$ which are expressed in the dimensionless units. The initial perturbation is set to $\delta B_{0 x}=0.024$. In order to avoid the complications of strong compressibility, we have set the sound speed to 4.0.

\subsection{Boundary conditions}

Our computational box has a grid of $256 \times 512 \times 256$ or for higher resolution runs, $512 \times 1024 \times 512$. In dimensionless units its size is $L_{x}=L_{z}=1$ and $L_{y}=2$. We double the size in the $\hat{y}$ direction to keep the driven turbulence away from the inflow boundary. There is no physical reason to do this, but driving turbulence near the inflow boundary produces numerical instabilities.

As mentioned earlier, we use three different types of boundary conditions, depending on the direction of the boundary: outflow boundary conditions along the $\hat{x}$ direction, inflow boundary conditions along the $\hat{y}$ direction and (sometimes) periodic boundary conditions along the $\hat{z}$ direction.

The open boundary conditions are the same as those used in our previous modelling. We refer to Kowal et al. (2009) for their detailed description. Briefly, we use simple "zerogradient" boundary conditions, setting the normal derivatives of the fluid variables (density and momentum) to zero. In the hydrodynamic limit this allows waves to leave the box without significant boundary reflections. In turbulence simulations this can lead to a slight drift in the fluid density. There is no requirement that the boundary density is constant, and inflows and outflows can cause a small net gain or loss from the system. Fortunately, changes in the total mass are small and only fluctuate around the initial value (Kowal et al., 2009). They do not influence our results significantly.

In order to incorporate the magnetic field into the open boundary conditions, we set the transverse components of the vector potential $\boldsymbol{A}$ using first order extrapolation. The normal derivative of the normal component is set to zero. In this way the normal derivatives of the transverse components of the magnetic field are zero, while the normal component of the magnetic field is calculated from the zero-divergence condition $\nabla \cdot \boldsymbol{B}=0$. This approach avoids the generation of non-zero magnetic divergence at the boundary. However, it has the drawback that it creates a small jump in the momentum flux across the boundary resulting from the presence of non-zero terms $\left(-B_{x}, B_{y}, B_{z}\right) \partial_{x} B_{x}$ at the $\mathrm{X}$ outflow boundary and $\left(B_{x},-B_{y}, B_{z}\right) \partial_{y} B_{y}$ at the $\mathrm{Y}$ inflow boundary. We have evaluated the velocity increment these terms produce at each time step. In models with the strongest turbulence, these terms were of the order of $10^{-6}$ and $10^{-8}$ at the $\mathrm{X}$ and $\mathrm{Y}$ boundaries, respectively. In the presence of strong outflows and inflows, generally of the order of unity, they are clearly negligible.

Simulations with explicit resistivity run into problems at the boundaries. In order to avoid a non-continuous resistive term and difficulties with the treatment of the current density $\boldsymbol{J}$ we have introduced a zone of decaying resistivity near the boundary. In a thin layer near the boundary, the value of resistivity $\eta_{u}$ decays down to a very small value chosen to be close to the numerical resistivity $\eta_{n}$ of our code. In our models, we adopt the value of $\eta_{n}=3 \cdot 10^{-4}$. None of this has an effect on the reconnection speeds. The validation of this method was presented in Kowal et al. (2009). 

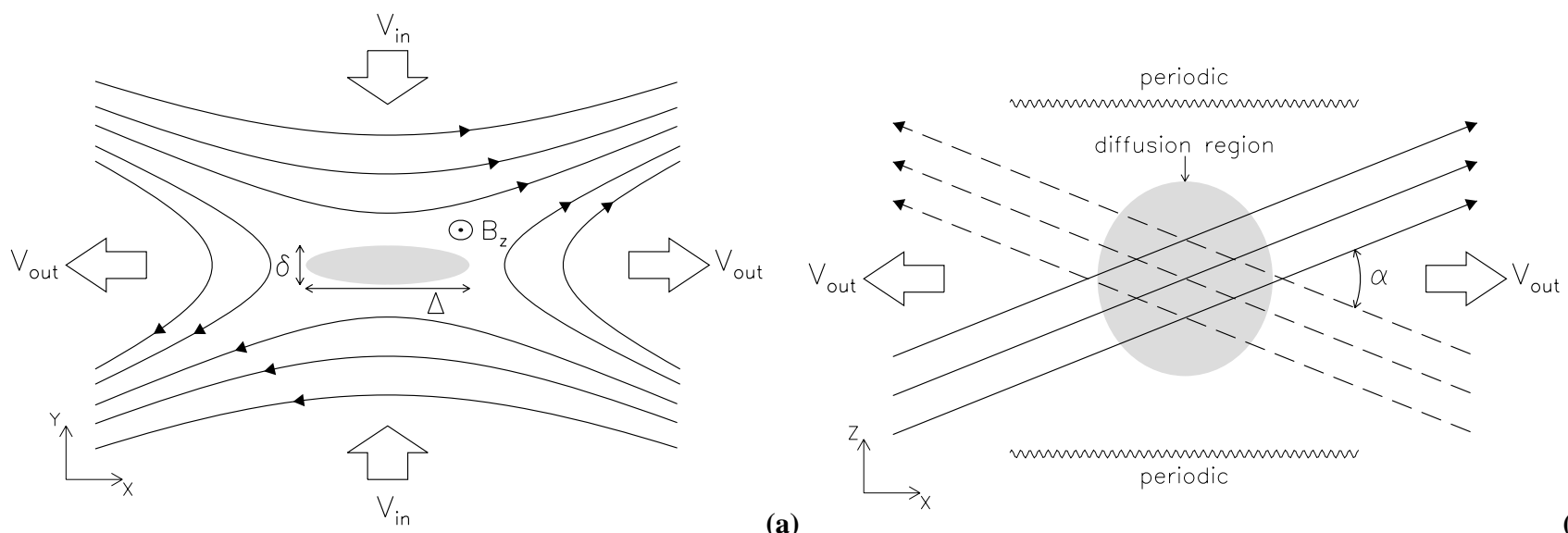

(b)

Fig. 2. A schematic of our magnetic field configuration projected on the XY (a) and XZ (b) planes. (a) XY projection of the magnetic field lines. The gray area describes the diffusion region where the incoming field lines reconnect. The longitudinal and transverse scales of the diffusion region are given by $\Delta$ and $\delta$, respectively. We use outflow and inflow boundary conditions in the $\hat{x}$ and $\hat{y}$ directions, respectively. (b) $\mathrm{XZ}$ projection of the magnetic field lines as seen from the top. Solid and dashed lines show the incoming field lines from the upper and lower parts of the domain, respectively. We see that the oppositely directed field lines are not anti-parallel but are set as an angle $\alpha$ determined by the strength of the shared component $B_{z}$. The $\hat{z}$ boundary conditions can be open or periodic, depending on the model (from Kowal et al., 2009).

\subsection{New Method of turbulence driving}

In our previous work we drove turbulence using a method described by Alvelius (1999), in which the driving term was implemented in the spectral space with discrete Fourier components concentrated around a wave vector $k_{\text {inj }}$ corresponding to the injection scale $l_{\text {inj }}=1 / k_{\text {inj }}$. We perturbed a number $N_{f}$ of discrete Fourier components of velocity in a shell extending from $k_{\text {inj }}-\Delta k_{\text {inj }}$ to $k_{\text {inj }}+\Delta k_{\text {inj }}$ with a Gaussian profile of the half width $k_{c}$ and the peak amplitude $\tilde{v}_{f}$ at the injection scale. The amplitude of driving is solely determined by its power $P_{\text {inj }}$, the number of driven Fourier components and the time step of driving $\Delta t_{f}$. The randomness in time makes the force neutral in the sense that it does not directly correlate with any of the time scales of the turbulent flow, and it also determines the power input solely by the force-force correlation.

On the right-hand side of Eq. (4), the forcing is represented by a function $\boldsymbol{f}=\rho \dot{\boldsymbol{u}}$, where $\rho$ is local density and $\dot{\boldsymbol{u}}$ is random acceleration calculated using the method described above. In a similar way, we can drive turbulence in the vector potential or magnetic field, which is represented by term $g$ on the right-hand side of the induction equation (Eq. 5).

In the new method of turbulence driving, we add individual eddies with random locations of their centres and random orientations, either to velocity or magnetic field, at random moments in time. This guarantees the randomness of new forcing.
Each eddy is calculated from a kernel function described by a directional vector $\boldsymbol{a}$ (with amplitude $|\boldsymbol{a}|$ ) multiplied by a Gaussian function

$\Psi(\boldsymbol{r})=\boldsymbol{a} \exp \left(-\frac{\left|\boldsymbol{r}-\boldsymbol{r}_{c}\right|^{2}}{2 \delta^{2}}\right)$,

where $\boldsymbol{r}_{c}$ is the location of the eddy centre and $\delta$ is the eddy width. An actual eddy is generated from such a kernel function by taking its curl, i.e., $\delta \boldsymbol{f}=\rho(\nabla \times \Psi) d t$ or $\delta \boldsymbol{g}=\nabla \times \Psi d t$ in the case of injection in velocity or magnetic field, respectively. For example, if we assume that we inject one eddy in the magnetic field at $\boldsymbol{r}_{c}=(0,0,0)$, and that the perturbing vector potential fluctuation has only the non-zero component, i.e., $\Phi=\left(0,0, \Phi_{z}\right)$, the contribution to magnetic field is expressed by

$\left[\begin{array}{l}\delta g_{x} \\ \delta g_{y} \\ \delta g_{z}\end{array}\right](x, y, z)=\frac{|\boldsymbol{a}|}{\delta^{2}} \exp \left(-\frac{|\boldsymbol{r}|^{2}}{2 \delta^{2}}\right)\left[\begin{array}{c}-y \\ x \\ 0\end{array}\right] d t$.

This function describes an eddy injected in the XY plane with the maximum amplitude $\delta g_{\max }=|\boldsymbol{a}| \delta^{-1} e^{-\frac{1}{2}}$ at the distance $r_{\max }=\delta$ and injection scale $l_{\text {inj }}=\delta$. We know the energy injected by one eddy, which is $\Delta E_{\text {eddy }}=\pi^{3 / 2}|\boldsymbol{a}|^{2} \delta / 2$, therefore, we can determine its amplitude $|\boldsymbol{a}|$ from the injection power $P_{\mathrm{inj}}$ and the injection rate $N_{\mathrm{inj}}$, which is the number of eddies injected in a time unit,

$P_{\text {inj }}=N_{\text {inj }} \Delta E_{\text {eddy }} \rightarrow|\boldsymbol{a}|=\sqrt{\frac{2 P_{\text {inj }}}{\pi^{3 / 2} N_{\text {inj }} \delta}}$.

These estimates are done for the 3-D case. In the 2-D case, the eddy energy is $\Delta E_{\text {eddy }}=\pi|\boldsymbol{a}|^{2} / 2$ and, therefore, the eddy amplitude can be determined from $|\boldsymbol{a}|=\sqrt{2 P_{\mathrm{inj}} /\left(\pi N_{\mathrm{inj}}\right)}$. 
In the new method there is no direct treatment of the velocity-force correlation, therefore, there is no guarantee that this correlation is zero and the injected power is completely determined by the force-force correlation. A reasonable solution to this problem would be to control the amount of injected energy and modify the amplitude of injected eddies or the injection rate at each time step in order to compensate the differences in the energy injected in the domain. The performed tests show, however, that although the velocityforce correlation is not zero, it is in fact fluctuating in time with a small amplitude and giving as a result a zero net contribution.

The new method drives turbulence directly in the real space, in contrast to the previous one, therefore, it can be applied locally. We drive turbulence in a sub-volume of the domain. The size of the sub-volume is determined by two scales, the radius $r_{d}$ on the XZ plane around the centre of the domain and the height $h_{d}$ describing the thickness of the driving region from the midplane. In this way, we avoid driving turbulence at the boundary and reduce the influence of driving on the inflow or outflow.

All models are evolved without turbulence for several dynamical times in order to allow the system to achieve stationary laminar reconnection. Then, at a given time $t_{b}$ we start driving turbulence by increasing its amplitude to the desired level, until $t_{e}$. In this way, we let the system adjust to a new state. From time $t_{e}$ the turbulence is driven with the full power $P_{\text {inj. }}$.

\subsection{Reconnection rate measure}

In the next sections, we measure the reconnection rate using the new method of reconnection rate measure introduced in Kowal et al. (2009) and described by a formula

$V_{\text {rec }}=\frac{1}{2\left|B_{x, \infty}\right| L_{z}}\left[\oint \operatorname{sign}\left(B_{x}\right) \boldsymbol{E} \cdot d \boldsymbol{l}-\partial_{t} \int\left|B_{x}\right| d A\right]$

where $B_{x}$ is the strength of reconnecting magnetic component, $\boldsymbol{E}$ is the electric field, $d \boldsymbol{A}$ is area element of an XZ plane across which we perform integration, $d \boldsymbol{l}$ is the line element separating two regions of the $\mathrm{YZ}$ plane defined by the sign of $B_{x},\left|B_{x, \infty}\right|$ is the asymptotic absolute value of $B_{x}$ and $L_{z}$ is the width of the box.

This method of the reconnection rate measure was derived from the magnetic flux conservation $\Psi$ and takes into account all processes contributing to the change of magnetic flux. The electric field $\boldsymbol{v} \times \boldsymbol{B}-\eta \boldsymbol{j}$ can be further divided into an advection term $\boldsymbol{v} \times B_{x} \hat{x}$, a shear term $\boldsymbol{v} \times\left(B_{y} \hat{y}+B_{z} \hat{z}\right)$, and a resistive term $-\eta j$. With this in mind the line integral can be rewritten as

$$
\begin{aligned}
& \oint \operatorname{sign}\left(B_{x}\right) \boldsymbol{E} \cdot d \boldsymbol{l}=\oint\left|B_{x}\right|\left(\boldsymbol{v}_{\perp} \times \hat{x}\right) \cdot d \boldsymbol{l} \\
& +\oint \operatorname{sign}\left(B_{x}\right) v_{x}\left(\hat{x} \times \boldsymbol{B}_{\perp}\right) \cdot d \boldsymbol{l}-\oint \eta \boldsymbol{j} \cdot d \boldsymbol{l} .
\end{aligned}
$$

Table 1. List of models.

\begin{tabular}{c|cccccc}
\hline Name & $B_{0 z}$ & $\eta_{u}\left[10^{-3}\right]$ & $v_{u}\left[10^{-3}\right]$ & $P_{\text {inj }}$ & $\Delta k_{\text {inj }}$ & Driving Type \\
\hline PD & 0.1 & 1.0 & 0.0 & 0.1 & 8 & old in $\boldsymbol{V}$ \\
& 0.1 & 1.0 & 0.0 & 0.2 & 8 & old in $\boldsymbol{V}$ \\
& 0.1 & 1.0 & 0.0 & 0.5 & 8 & old in $\boldsymbol{V}$ \\
& 0.1 & 1.0 & 0.0 & 1.0 & 8 & old in $\boldsymbol{V}$ \\
& 0.1 & 1.0 & 0.0 & 2.0 & 8 & old in $\boldsymbol{V}$ \\
& 1.0 & 1.0 & 0.0 & 0.1 & 8 & old in $\boldsymbol{V}$ \\
& 1.0 & 1.0 & 0.0 & 0.2 & 8 & old in $\boldsymbol{V}$ \\
& 1.0 & 1.0 & 0.0 & 0.5 & 8 & old in $\boldsymbol{V}$ \\
& 1.0 & 1.0 & 0.0 & 1.0 & 8 & old in $\boldsymbol{V}$ \\
$\rightarrow$ & 1.0 & 1.0 & 0.0 & 2.0 & 8 & old in $\boldsymbol{V}$ \\
$\rightarrow$ & 0.1 & 1.0 & 0.0 & 0.2 & 8 & new in $\boldsymbol{B}$ \\
$\rightarrow$ & 0.1 & 1.0 & 0.0 & 0.5 & 8 & new in $\boldsymbol{B}$ \\
$\mathrm{HR}$ & 0.1 & 1.0 & 0.0 & 1.0 & 8 & new in $\boldsymbol{B}$ \\
& 0.1 & 0.5 & 0.0 & 1.0 & 8 & new in $\boldsymbol{V}$ \\
\hline $\mathrm{SD}$ & 0.1 & 1.0 & 0.0 & 1.0 & 5 & old in $\boldsymbol{V}$ \\
& 0.1 & 1.0 & 0.0 & 1.0 & 8 & old in $\boldsymbol{V}$ \\
& 0.1 & 1.0 & 0.0 & 1.0 & 12 & old in $\boldsymbol{V}$ \\
& 0.1 & 1.0 & 0.0 & 1.0 & 16 & old in $\boldsymbol{V}$ \\
& 0.1 & 1.0 & 0.0 & 1.0 & 25 & old in $\boldsymbol{V}$ \\
& 1.0 & 1.0 & 0.0 & 1.0 & 5 & old in $\boldsymbol{V}$ \\
& 1.0 & 1.0 & 0.0 & 1.0 & 8 & old in $\boldsymbol{V}$ \\
& 1.0 & 1.0 & 0.0 & 1.0 & 12 & old in $\boldsymbol{V}$ \\
& 1.0 & 1.0 & 0.0 & 1.0 & 16 & old in $\boldsymbol{V}$ \\
$\rightarrow$ & 1.0 & 1.0 & 0.0 & 1.0 & 25 & old in $\boldsymbol{V}$ \\
$\rightarrow$ & 0.1 & 1.0 & 0.0 & 1.0 & 8 & new in $\boldsymbol{B}$ \\
$\mathrm{HR}$ & 0.1 & 1.0 & 0.0 & 1.0 & 24 & new in $\boldsymbol{B}$ \\
& 0.1 & 1.0 & 0.0 & 1.0 & 32 & new in $\boldsymbol{B}$ \\
$\mathrm{VD}$ & 0.1 & 0.5 & 0.0 & 1.0 & 8 & new in $\boldsymbol{V}$ \\
& 0.1 & 1.0 & 0.2 & 1.0 & 8 & old in $\boldsymbol{V}$ \\
& 0.1 & 1.0 & 0.5 & 1.0 & 8 & old in $\boldsymbol{V}$ \\
& 0.1 & 1.0 & 1.0 & 1.0 & 8 & old in $\boldsymbol{V}$ \\
& 0.1 & 1.0 & 2.0 & 1.0 & 8 & old in $\boldsymbol{V}$ \\
& 0.1 & 1.0 & 3.0 & 1.0 & 8 & old in $\boldsymbol{V}$ \\
& 0.1 & 1.0 & 4.0 & 1.0 & 8 & old in $\boldsymbol{V}$ \\
& 0.1 & 1.0 & 5.0 & 1.0 & 8 & old in $\boldsymbol{V}$ \\
\hline & & & & & & \\
& & & & & \\
& & & & & &
\end{tabular}

This new reconnection measure contains the time derivative of the absolute value of $B_{x}$, and a number of boundary terms, such as advection of $B_{x}$ across the boundary and the boundary integral of the resistive term $\eta j$. The additional terms include all processes contributing the time change of $\left|B_{x}\right|$. In particular, they can have non-zero values.

\subsection{Table of simulated models}

In Table 1 we list parameters of all the models presented in this paper including models from Kowal et al. (2009) and models with new driving. As in the previous paper, we divided them into several groups. In each group, we calculated models in order to study the dependence of the reconnection rate on a characteristic parameter of turbulence or resistivity. We have studied the dependence of reconnection on the power of turbulence (models "PD"), injection scale (models "SD") and viscosity (models "VD"). Models with new driving are marked with a right arrow $(\rightarrow)$, and models with new driving and higher resolution are marked with a symbol "HR".

Only the varying parameters are listed in the table, the strength of guide field $B_{0 z}$, the uniform resistivity $\eta_{u}$, the 


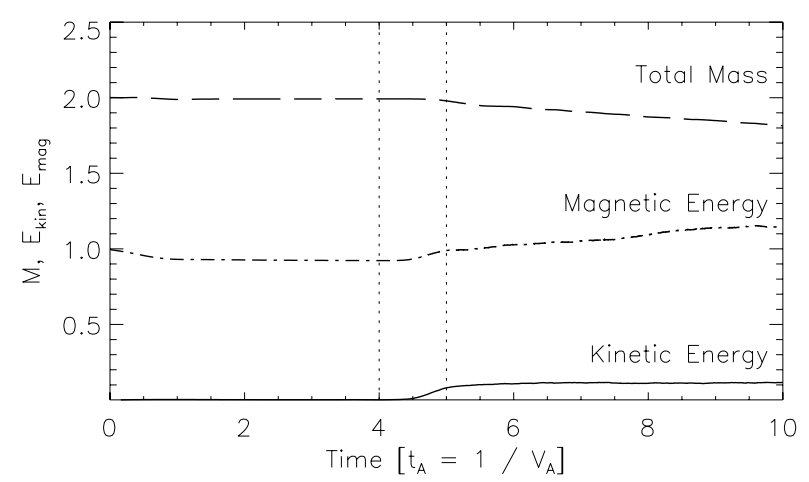

Fig. 3. Evolution of total mass $M$ and kinetic and magnetic energies $E_{\text {kin }}$ and $E_{\text {mag }}$, respectively. Two dotted vertical lines bound the period of gradually increasing turbulence. The resistivity in this model is set to $\eta=10^{-3}$ and the shared component of magnetic field $B_{0 z}=0.1$. In this model, we inject turbulence in the magnetic field.

uniform viscosity $v_{u}$, the power of turbulence $P_{\text {inj }}$ and its injection scale $k_{\text {inj }}$, and the method of turbulence driving.

All models presented in this section were calculated with the grid size $\Delta x \approx 0.004$ corresponding to the resolution $256 \times 512 \times 256$, except the model marked with symbol "HR", which was simulated with the resolution $512 \times 1024 \times$ $512(\Delta x \approx 0.002)$.

\section{Results}

\subsection{Time evolution of energies and reconnection rate}

In Fig. 3, we present an example of the evolution of total mass and kinetic and magnetic energies in a model with $P_{\text {in }}=1.0, k_{f}=8$ and $\eta_{u}=10^{-3}$. We inject turbulence into the magnetic field using the new forcing method, gradually increasing its strength from $t=4$ to $t=5$. This period is marked by two dotted vertical lines in Fig. 3. We see an increase of kinetic energy during this period due to the injection and saturation after $t=5$. The kinetic energy preserves constant value during the turbulent stage very well. The magnetic energy increases during this stage, slowly saturating. This increase is attributed to the injection of magnetic eddies. On the contrary, the total mass in the system decays slowly. We emphasize that since we use open boundary conditions, not perfect conservation of mass and energies, it is possible in the presence of turbulence.

In Fig. 4, we show the evolution of reconnection rates $V_{\text {rec }}$ for two models with the same set of initial conditions, but in the first model we drove turbulence by injecting magnetic eddies using the new method described in this paper (black line), and in the second model we inject velocity fluctuations using the old method described in Kowal et al. (2009) (blue line). In this plot, we recognise an increase of both rates

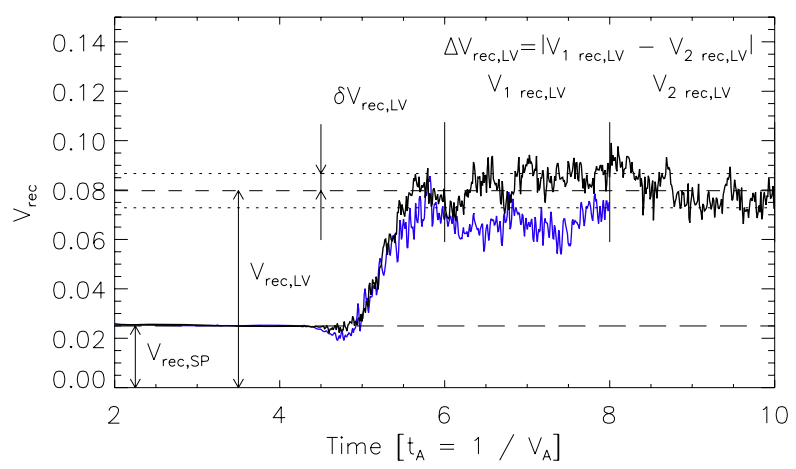

Fig. 4. Evolution of the reconnection rate $V_{\text {rec }}$ (black) for the same model as in Fig. 3. Blue line shows the evolution of reconnection in a model with the same parameters in which the turbulence were driven using the old method. In this plot, we present the measured rates of the Sweet-Parker reconnection $V_{\text {rec,SP }}$ and during the presence of turbulence, $V_{\text {rec,LV. Symbol } \delta} V_{\text {rec,LV }}$ is the time variance. $\Delta V_{\text {rec, } \mathrm{LV}}$ is the estimated uncertainty of the measure.

during the introduction of turbulence. After the transition period between $t=4$ and $t=5$, when the system adjusts to a new state, both measures coincide and even though they fluctuate, they reach a stationary state characterised by faster reconnection. Both types of turbulence bring the reconnection rate to a similar level. A somewhat higher reconnection rate in the model with new driving could be attributed to the fact that this model was calculated using the 5th order spatial reconstruction and the 3 rd order integration in time, in contrast to the old model where we used the second order methods. Lower order, especially in the spatial interpolation, introduces additional numerical diffusion decreasing the amplitudes of turbulent fluctuations at scales comparable to the current sheet scale.

In Fig. 4, we also show the way of measuring the reconnection rates, in the Sweet-Parker and LV99 stages, $V_{\text {rec,SP }}$ and $V_{\text {rec,LV }}$, respectively. Because the reconnection rates fluctuate in the presence of turbulence, we also measure their time variance $\delta V_{\text {rec, LV }}$ using the standard deviation. In addition to the time variance of $V_{\mathrm{rec}}$, we measure their errors by splitting the averaging region into two subregions and after averaging the rates $V_{1 \text { rec }}$ and $V_{2 \text { rec }}$ over each subregion (see Fig. 4), we take the absolute value of their difference $\Delta V_{\text {rec }}=V_{1 \text { rec }}-V_{2 \text { rec }}$. This difference corresponds to the error of $V_{\text {rec }}$, i.e., it is different from zero if the rate is not constant in time. In all further analysis and presented plots, we use values estimated in this way. These measures correspond exactly to those presented in Kowal et al. (2009).

\subsection{Topology of magnetic field}

In this section, we compare field topologies in two example models run with the same set of parameters, but with different types of driving. Both models have been simulated 
with the uniform resistivity $\eta_{u}=10^{-3}$ and the resolution $256 \times 512 \times 256$. We injected turbulence with power $P_{\text {inj }}=1.0$ at the injection scale $k_{\text {inj }}=8$. The only difference between models is the way we injected turbulence. In the old model, we inject velocity fluctuations with random phases in Fourier space and then transform them to real space and shape by a window in order to limit the injection to the specified region near the current sheet. In the new model, we inject magnetic loops with random locations and random orientations in the 3-D volume near the current sheet. The way of injecting turbulence is essentially different in both cases.

In Fig. 5, we show examples of XY-cuts (upper row) and XZ-cuts (lower row) through the box for the model with old driving. In the left and middle columns, we show topologies of the velocity and magnetic field, respectively, with the intensities corresponding to the amplitude of components parallel to the plotted plane. In the right column, we show the absolute value of current density with overplotted magnetic vectors. Velocity has a very complex and mixed structure near the midplane due to constant injection of fluctuations in this region (see the left panel in Fig. 5). The majority of the velocity fluctuations is perpendicular to the mean magnetic field. This is because we are in the nearly incompressible regime of turbulence (large plasma $\beta$ ) and most of the fluctuations propagate as Alfvén waves along the mean magnetic field. Slow and fast waves, whose strengths are significantly reduced, are allowed to propagate in directions perpendicular to the mean field as well. As a result, a big fraction of the turbulent kinetic energy leaves the box along magnetic lines. We observe, however, an efficient bending of magnetic lines at the midplane where the field is weaker (see the upper middle plot in Fig. 5). This is not the result of a driving, but result of reconnection. In general the interface between positively and negatively directed magnetic lines is much more complex than in the case of Sweet-Parker reconnection. This complexity favours creation of enhanced current density regions, where the local reconnection works faster (see the right panel of Fig. 5). Since we observe multiple reconnection events happening at the same time, the global reconnection rate should be significantly enhanced.

In Fig. 6, we show similar examples of XY-cuts (upper row) and XZ-cuts (lower row) through the box, but for a model with the new way of driving turbulence. Here, a big number of individual eddies is injected in the magnetic field with random locations and random orientations in domain. Comparing to plots in Fig. 5, we see differences but also some clear similarities. Among the similarities, we note a highly turbulent region near the current sheet seen in all XY-cuts, with the current sheet itself strongly deformed and fragmented into many small scale current sheets (the right column of Figs. 5 and 6). We see also some small increase of magnetic field strength near the current sheet (middle top panels) resulting from working turbulence in the injection region. Among the differences we can list somewhat different distributions of the fragmented current sheets in the new model with clear enhancements in the locations where the magnetic eddies are injected at that moment. These enhancements are clearly seen in the magnetic topology and current density plots (middle and right columns). In order to decrease those strong disturbances of the magnetic lines, we shall reproduce the same model with higher injection rate and reduced amplitudes of individual eddies. Another difference is the strength of current density $\boldsymbol{J}$. In the model with old driving, we see more volume in which $|\boldsymbol{J}|$ reaches high magnitude and its structure is elongated with the local field. In the model with new driving, the current density with high strength seems to be less correlated with the local field, probably due to the presence of newly injected eddies. In the intermediate strengths, the structure of $|\boldsymbol{J}|$ seems to be better correlated with the local field.

We see from this comparison that models with different driving of turbulence demonstrate different topologies of the fields. In the next sections, we show that the averaged reconnection rates do not change significantly, confirming that the way we inject turbulence is of less importance and only its strength and injection scale have influence on $V_{\text {rec }}$.

\subsection{Dependence on turbulence strength}

Models with the new method of turbulence driving are listed in Table 1. We run a few models with varying turbulent powers in order to verify if the new driving modifies our previous results. In these models, we kept the same parameters as in the previous ones which allowed us to confirm the dependence of the reconnection rate $V_{\text {rec }}$ on the power of injected turbulence $P_{\text {inj }}$.

Figure 7 shows the values of reconnection speed $V_{\text {rec }}$ in models with turbulent power $P_{\text {inj }}$ varying in the range of values by more than one order of magnitude, from 0.1 to 2.0, for all previously shown models (black symbols) in Kowal et al. (2009) and for new models (blue and red symbols) in which we drove turbulence using the new method. Because the evolution of $V_{\text {rec }}$ in new models reaches stationarity after time $t=6$, we averaged $V_{\text {rec }}$ from $t=6$ to $t=10$ in these models. Figure 4 shows that the reconnection rates oscillate around their mean values. In Fig. 7, we plot how the averaged reconnection speed depends on the strength of turbulence. Filled symbols represent the averaged reconnection rate in the presence of turbulence. The dotted line corresponds to the reconnection rate during the Sweet-Parker process, i.e., without turbulence. The error bars show the time variance of $V_{\text {rec. }}$. The size of symbols indicates the uncertainty in our estimate of the reconnection speed $\Delta V_{\text {rec, LV nor- }}$ malized to the uncertainty in the reconnection speed during

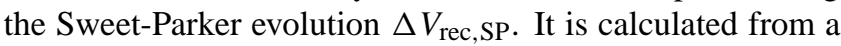
formula size $=2.0-\ln \Delta V_{\mathrm{rec}, \mathrm{LV}} / \ln \Delta V_{\mathrm{rec}, \mathrm{SP}}$. If $\Delta V_{\mathrm{rec}, \mathrm{LV}}$ is of the order of $\Delta V_{\text {rec,SP }}$ their symbols have the same sizes.

The reconnection rates for models with new driving, which is described in Sect. 3.4, confirm the theoretical dependence of $V_{\text {rec }}$ on the injected power, which scales as $\sim P_{\text {inj }}^{1 / 2}$. There 

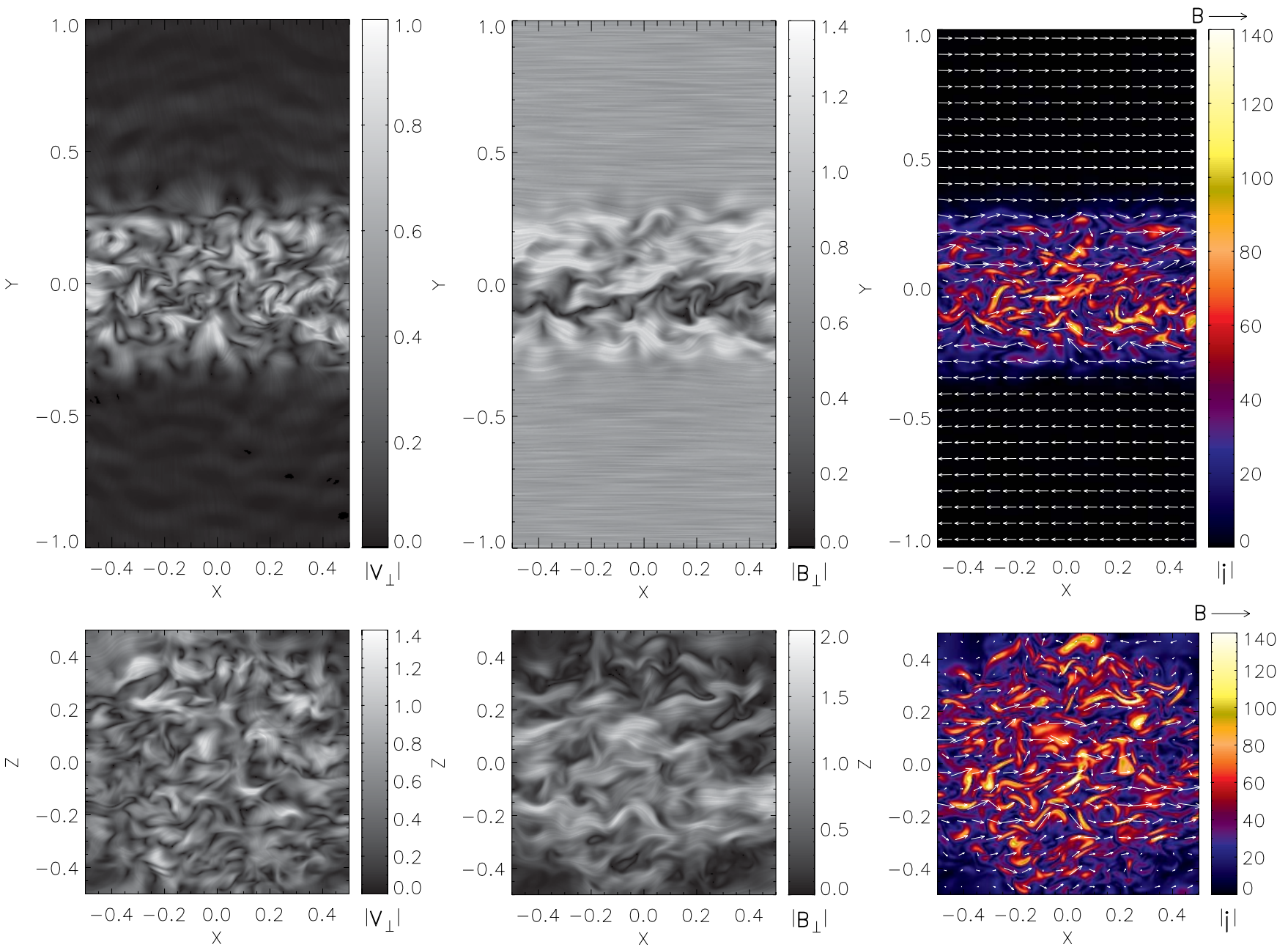

Fig. 5. Topology and strength of the velocity field (left panel) and magnetic field (middle panel) in the presence of fully developed turbulence for an example model with old driving at time $t=12$. In the right panel, we show distribution of the absolute value of current density $|\boldsymbol{J}|$ overlapped with the magnetic vectors. The images show the XY-cut (upper row) and XZ-cut (lower row) of the domain at the midplane of the computational box. Turbulence is injected with power $P_{\text {inj }}=1$ at scale $k_{\text {inj }}=8$ into velocity. Magnetic field reversals observed are due to magnetic reconnection rather than driving of turbulence, which is sub-Alfvénic.

is no significant difference between models in which turbulence was driven in velocity and in magnetic field. This is in agreement with the LV99 prediction, that the reconnection rate does not depend on the type of turbulence.

\subsection{Dependence on injection scale}

The reconnection rate $V_{\text {rec }}$ in the presence of turbulence depends only on the strength of turbulence and its injection scale $l_{\text {inj }}$, according to Eq. 1, for a fixed magnitude of the anti-parallel magnetic field component. In the previous subsection, we presented studies on the turbulent power dependence. In this subsection, we aim to study the injection scale dependence. For this purpose, we performed several models with the new way of driving turbulence as well, in order to verify if they confirm the dependence of the reconnection speed $V_{\text {rec }}$ on the scale $l_{\text {inj }}$ at which we inject turbulence. The new models are listed in Table 1 . We inject turbulence at several scales, from $k_{\text {inj }}=8$ to $k_{\text {inj }}=32$. At the upper end of this range, the turbulence barely broadens the Sweet-Parker current sheet. At the lower end the turbulent eddies are barely contained within the volume in which we excite turbulent motions.

In Fig. 8, we present the reconnection speed dependence on the injection scale. We plot the averaged $V_{\text {rec }}$ for old models (black symbols) completed by the values from new models with alternative driving (blue and red symbols). From the plot we clearly see a strong dependence of the reconnection rate on the injection scale. The new models very precisely follow the same dependence, confirming again that the type of turbulent driving has no influence on the process of reconnection, and only the power and injection scale of this driving have strong importance. Similarly, as in the 

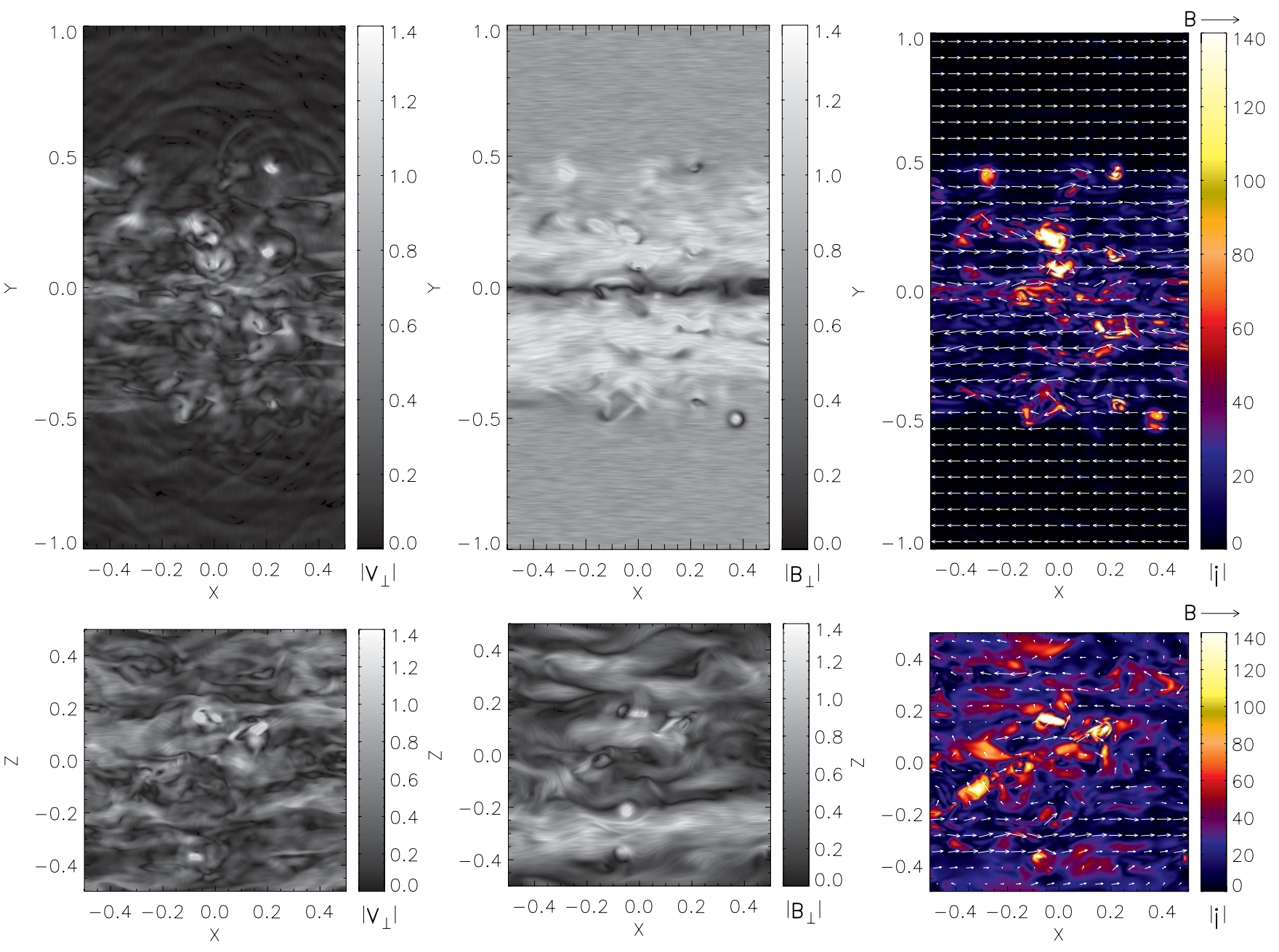

Fig. 6. Topology and strength of the velocity field (left panel) and magnetic field (middle panel) in the presence of fully developed turbulence for an example model with new driving at time $t=10$. In the right panel, we show distribution of the absolute value of current density $|\boldsymbol{J}|$ overlapped with the magnetic vectors. The images show the XY-cut (upper row) and XZ-cut (lower row) of the domain at the midplane of the computational box. Turbulence is injected with power $P_{\text {inj }}=1$ at scale $k_{\text {inj }}=8$ directly in the magnetic field.

power dependence plot, the new models have slightly higher reconnection speeds comparing to the old ones. This is due to reduced numerical dissipation of velocity, since in the new models we used higher order methods. Dissipation removes energy at small scales. If it is smaller, due to higher order numerical scheme, the turbulent fluctuations reach higher amplitudes at the current sheet scale. This influences the rate of individual reconnection events improving slightly the global reconnection rate $V_{\text {rec }}$.

Figure 8 shows a bit weaker scaling with the injection scale than that predicted by LV99 model, i.e., $V_{\text {rec }} \sim l_{\text {inj. }}$. We see several possible sources for the discrepancy. For instance, the existence of a turbulent inverse cascade can modify the effective $l_{\text {inj. }}$. In addition, reconnection can also modify the characteristics of turbulence, such as the power spectrum and anisotropy. We aim to study these problems in future work.

\subsection{Dependence on viscosity}

In Kowal et al. (2009) we performed studies of the reconnection rate on the resistivity, both the uniform and anomalous ones, and we obtained great agreement with the SweetParker scaling $V_{\text {rec }} \sim \eta_{u}^{1 / 2}$ for the case without turbulence, and no dependence on resistivity in the presence of turbulence, as was predicted in LV99. In this section, we performed additional studies of the reconnection rate dependence on viscosity. The dissipation scale of turbulent cascade is related to the magnitude of viscosity. If the dissipation works at scales larger than the current sheet thickness, the turbulence cascade stops before reaching the current sheet and the global reconnection rate should be reduced. The reconnection will still be enhanced by the broadened ejection region, allowing for more efficient removal of the reconnected magnetic flux. 


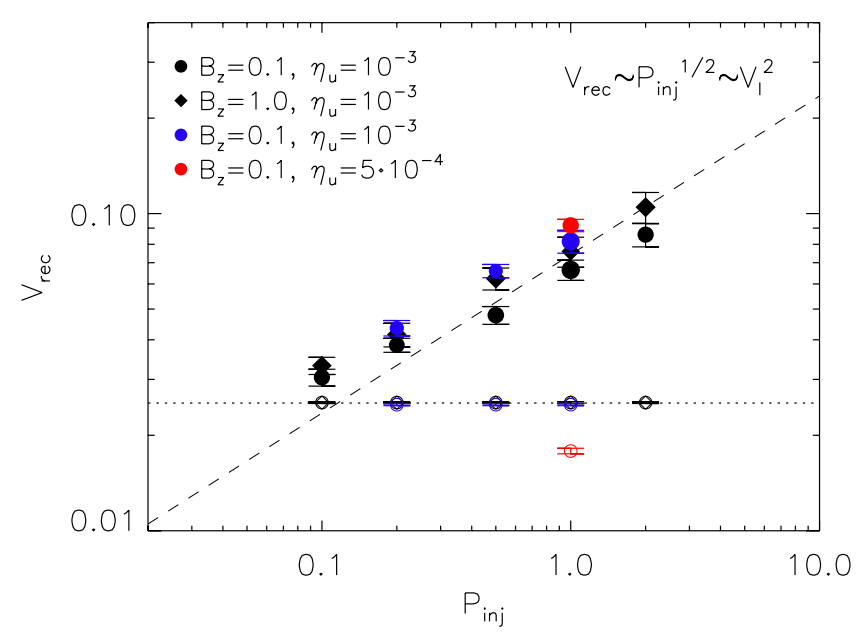

Fig. 7. The dependence of the reconnection speed $V_{\text {rec }}$ on $P_{\text {inj }}$ updated by symbols from new models. Blue symbols show models with new driving in which the eddies where injected in magnetic field instead of velocity, as in the previous models (black symbols). The dotted line corresponds to the Sweet-Parker reconnection rate for models with $\eta_{u}=10^{-3}$. A unique red symbol shows the reconnection rates from model with new driving in velocity performed with higher resolution $(512 \times 1024 \times 512)$ and resistivity coefficient reduced to $\eta_{u}=5 \cdot 10^{-4}$. Error bars represent the time variance of $V_{\text {rec }}$. The size of symbols corresponds to the error of $V_{\text {rec }}$ (the way we calculate errors is described in Sect. 4.1).

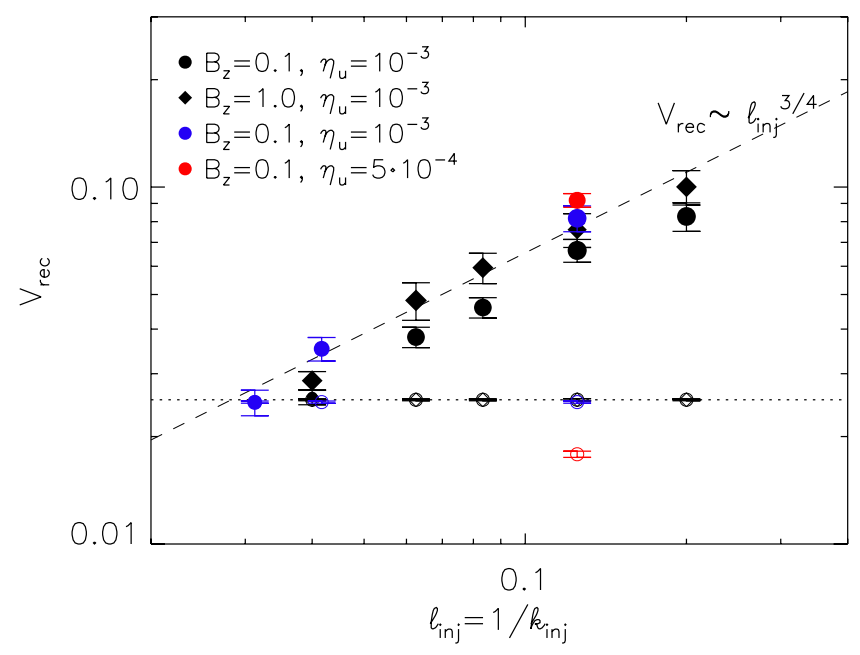

Fig. 8. The dependence of the reconnection speed $V_{\text {rec }}$ on $l_{\text {inj }}$ with additional models in which turbulence was driven in a new way, as described in Sect. 3.4. Similarly to Fig. 7, blue symbols show models with perturbed magnetic field, and red symbols correspond to a high resolution model with reduced uniform resistivity in which turbulence was driven in velocity. The dotted line corresponds to the Sweet-Parker reconnection rate for models with $\eta_{u}=10^{-3}$. Error bars and the size of symbols have the same meaning as in Fig. 7.

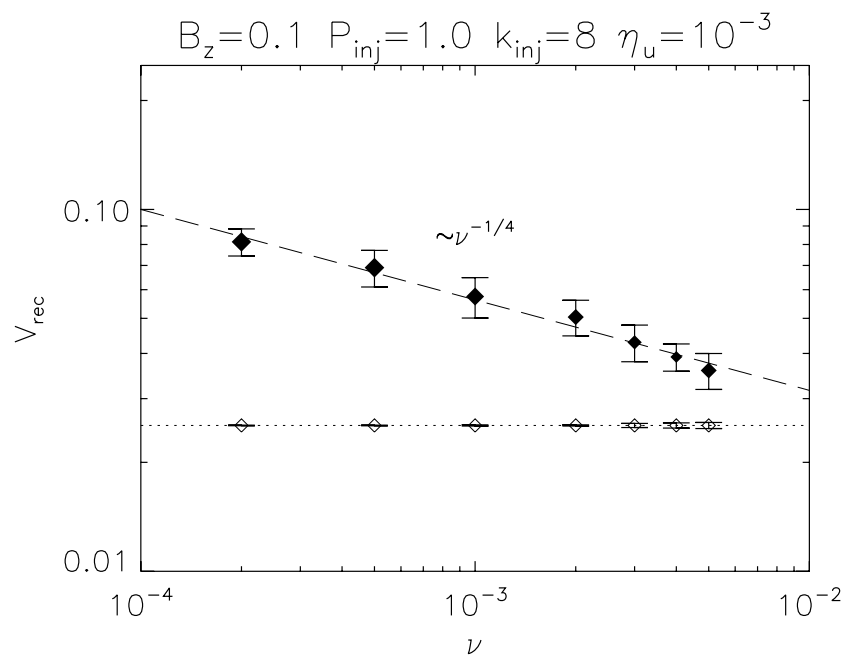

Fig. 9. The dependence of the reconnection speed $V_{\text {rec }}$ on the uniform viscosity coefficient $v$. As explained in the text, the reconnection speed is reduced with increasing value of $v$. The dotted line corresponds to the Sweet-Parker reconnection rate. Error bars and the size of symbols have the same meaning as in Fig. 7.

In Fig. 9, we show reconnection rates for models with varying viscosity coefficient. Although there is not prediction for this dependence in the LV99 model, we could test it numerically. In the Fig. 9, we see a weak dependence $V_{\text {rec }} \sim v^{-1 / 4}$. This dependence might be also useful in understanding the reconnection speed differences between models with the same set of parameters but different resolutions, or solved with different orders of the numerical scheme. At low resolutions or low order schemes, the numerical viscosity is expected to be larger, thus we should observe reduced reconnection speeds in those cases. This is confirmed in Figs. 7 and 8 where we compare old models done with the second order scheme, and new models done with higher order schemes and higher resolutions. In those plots all new models demonstrate slightly higher reconnection rates.

\section{Discussion}

\subsection{LV99 in collisional and collisionless plasma}

The LV99 model was introduced for both collisional and collisionless media and it claimed that the microphysics of collisionless reconnection events does not change the resulting reconnection rates. This point was subjected to further scrutiny in Eyink et al. (2011) who provided a thorough investigation of the problem and concluded that for most of astrophysical collisionless plasmas the LV99 model should be applicable, provided that plasma is turbulent. With turbulence being ubiquitous in astrophysical conditions, this hardly constraints the applicability of the LV99 model. 
The LV99 model of reconnection is applicable to the collisional medium, such as the ISM, which is both turbulent and magnetized, and where the Hall-MHD reconnection does not work (Yamada, 2007). For instance, for Hall-MHD reconnection to be applicable, it is required that the Sweet-Parker current sheet $\delta_{\mathrm{SP}}$ width is smaller than the ion inertial length $d_{i}$. Thus, the "reconnection criterion for media to be collisionless" is $\left(L / d_{i}\right)^{1 / 2} /\left(\omega_{c} \tau_{e}\right)<1$, which presents a more severe constraint on the possible rate of collisions. As a result, magnetic reconnection happens to be mediated by the Hall-MHD only if the extend of the contact region $L$ (see Fig. 1) does not exceed $10^{12} \mathrm{~cm}$. Magnetic fields in the ISM should interact over much larger scales.

The LV99 model works in astrophysical environments to which the Hall-MHD reconnection is applicable, as well, like Solar corona, interplanetary medium, if the level of turbulence is high enough. The reconnection on microscales can happen fast, i.e., in the Hall-MHD fashion. This may not change, however, the global reconnection rate. The LV99 model shows that even with relatively slow Sweet-Parker reconnection at microscales the global reconnection is limited not by Ohmic resistivity, but by the rate of magnetic field wondering. We believe that the Hall-MHD local reconnection of magnetic fields is taking place in the interplanetary medium, which is being tested by local in situ measurements, while the global reconnection rates are determined by magnetic field wandering as prescribed in LV99.

\subsection{Limitations of 2-D reconnection}

In the absence of a quantitative model to be tested, simulations aimed at studying the reconnection speed have been done in 2-D, both for collisional and collisionless regimes. This allowed achieving higher resolutions (compared to those contemporary available in 3-D), but substantially constrained magnetic field dynamics. For instance, the closest study to ours was done by Matthaeus and Lamkin (1985) (see also Matthaeus and Lamkin, 1986). The authors studied 2-D magnetic reconnection in the presence of external turbulence. An enhancement of the reconnection rate was reported, but the numerical setup precluded the calculation of a long-term average reconnection rate. A more recent study along the approach of Matthaeus and Lamkin (1985) is one in Watson et al. (2007), where the effects of small scale turbulence on 2-D reconnection were studied and no significant effects of turbulence on reconnection were reported for the setup chosen by the authors. Later, Servidio et al. (2010) redid the modelling of 2-D turbulent reconnection following Matthaeus and Lamkin (1985) with much higher resolutions. They used an advanced technique to detect precisely all Xpoints in the domain and then performed statistical studies confirming the Sweet-Parker relation for the reconnection rate as a function of X-point geometry. The development of different techniques to study magnetic reconnection is very important. Even though their modelling was limited to one type of highly super-Alfvénic decaying turbulence (the initial uniform magnetic field was zero), they reported reconnection rates with normalized values $0.1-0.3$ and confirmed the importance of turbulence for modifying the character of magnetic reconnection and specifies heating and transport as the effect of particular significance, as well as formation of Petschek-type "X-points" in 2-D turbulence. Due to the lack of large scale magnetic field configuration, their model represents a specific case, far from the generic situation observed in the astrophysical objects where the mean and turbulent components of magnetic fields have comparable strengths. Therefore, these studies cannot predict the global reconnection rate, as well. Moreover, Servidio et al. (2010) interpreted successful numerical confirmation of the LV99 model as a result of strong turbulence, although Kowal et al. (2009) addressed this problem carefully showing that the amplitudes of velocity fluctuations, both injected and obtained from spectra of developed and stationary turbulence, are fractions of Alfvén speed.

The fact that our study is in 3-D is essential, as the LV99 model is intrinsically three dimensional. The general picture is of tangled field lines with reconnection taking place via a series of "Y-points" or modified Sweet-Parker sheets distributed in some fractal way throughout the turbulence. A large scale Sweet-Parker sheet will be replaced by a more fractured surface, but the current sheets will occupy a vanishingly small fraction of the total volume and the field reversal will remain relatively well localized. The model predicts that the reconnection speed would be approximately equal to the strong turbulent velocity with a modest dependence on the ratio of the eddy length to the current sheet length. There should be no dependence on resistivity. The major results contained in our figures showing the dependence of the reconnection speed on resistivity, input power and input scale agree with the quantitative predictions of the LV99 model. We are not aware of any competing models to compare our simulations with.

The major differences from the present study stem from the fact that we test a 3-D model of reconnection, as the LV99 depends on effects, like field wandering, that happen only in 3-D. In order to show how different 2-D and 3-D worlds are, we performed similar studies to those presented in Kowal et al. (2009), but limiting the domain to two dimensions (see Kulpa-Dybeł et al., 2010). In Kulpa-Dybeł et al. (2010), we demonstrated that 2-D magnetic reconnection in the presence of turbulence depends on the Ohmic resistivity, therefore, it is not fast. Also, the dependencies on the turbulent power and injection scales were significantly weaker than in the LV99. This dependence of 2-D reconnection rate on Ohmic resistivity in the presence of turbulence, although weaker than the Sweet-Parker relation $V_{\mathrm{rec}} \sim \eta^{-1 / 2}$, has been independently confirmed by Loureiro et al. (2009) studies, performed with a very different approach. These differences call for deliberation with a simple extension of conclusions coming from 
the 2-D modelling to a natural for magnetic field fully three dimensional world.

\subsection{Applications of the LV99 model}

Reconnection is one of the most fundamental processes involving magnetic fields in conducting fluids or plasmas. Therefore, the identification of a robust process responsible for reconnection has many astrophysically important consequences. Below we list a few selected implications of the successful validation of the LV99 model.

Numerical studies on Fermi acceleration in turbulent reconnection have a long history (e.g. Matthaeus et al., 1984; Goldstein et al., 1986; Ambrosiano et al., 1988; Drake et al., 2006; Hoshino, 2012). In the Sweet-Parker model, it has been shown that particles can accelerate due to the induced electric field in the reconnection zone (Litvinenko, 2003). This one - shot acceleration process, however, is constrained by the narrow thickness of the acceleration zone which has to be larger than the particle Larmor radius and by the strength of the magnetic field. Therefore, the efficiency of this process is rather limited. Besides, it also does not predict a power-law spectrum, as generally observed for cosmic rays. Observations have always been suggestive that magnetic reconnection can happen at high speed in some circumstances, in spite of the theoretical difficulties in explaining it. For instance, the phenomenon of solar flares suggests that magnetic reconnection should be first slow in order to ensure the accumulation of magnetic flux and then suddenly become fast in order to explain the observed fast release of energy. The LV99 model can naturally explain this and other observational manifestations of magnetic reconnection. Consider a particle entrained on a reconnected magnetic field line (see Fig. 1). This particle may bounce back and forth between magnetic mirrors formed by oppositely directed magnetic fluxes moving towards each other with the velocity $V_{\text {rec }}$. Each bounce will increase the energy of a particle in a way consistent with the requirements of the first-order Fermi pro$\operatorname{cess}^{2}$ (de Gouveia Dal Pino and Lazarian, 2001, 2003; de Gouveia dal Pino and Lazarian, 2005; Lazarian, 2006). This is in contrast to the second-order Fermi acceleration that is frequently discussed in terms of accelerating particles by turbulence generated by reconnection (La Rosa et al., 2006). The numerical studies of the particle acceleration supporting these ideas have been already started (Kowal et al., 2011a,b). An interesting property of this acceleration mechanism is that it is also potentially testable observationally, since the resulting spectrum of accelerated particles is different from

\footnotetext{
${ }^{2}$ Another way of understanding the acceleration of energetic particles in the reconnection process above is to take into account that the length of magnetic field lines is decreasing during reconnection. As a result, the physical volume of the energetic particles entrained on the field lines is shrinking. Thus, due to Liouville's theorem, their momentum should increase to preserve the constancy of the phase volume.
}

that arising from a shock. de Gouveia Dal Pino and Lazarian (2001); de Gouveia dal Pino and Lazarian (2005) used this mechanism of particle acceleration to explain the synchrotron power-law spectrum arising from the flares of the microquasar GRS 1915+105.

Further applications can be found in solar physics. Following Zweibel and Yamada (2009), we note that solar flares inspired much of the earlier research on reconnection (see Pneuman, 1981; Bastian et al., 1998). As the plasma involved is substantially rarefied, the restrictive conditions for the collisionless reconnection are satisfied in this particular environment. Cassak et al. (2005) stated that bistable Hall reconnection can be important in this case. Stochastic reconnection provides an alternative explanation. Indeed, an important prediction of the LV99 model is related to the reconnection instability that arises in the situation when the initial structure of the flux prior to reconnection is laminar. Reconnection at the Sweet-Parker rate is negligible. This allows magnetic flux to accumulate. However, when the degree of stochasticity exceeds a threshold value, the reconnection itself should excite more turbulence, creating a positive feedback resulting in a flare (see Lazarian and Vishniac, 2009). The instability is a generic property of laminar field reconnection in both collisionless and collisional environments. Referring to the Sun, one may speculate that the difference between gradual and eruptive flares arises from the original state of magnetic field prior to the flare, at least in some specific situations. In the case when the magnetic field is sufficiently turbulent the accumulation of magnetic flux does not happen and the flare is gradual. Similarly, the observed spatial spread of energy release during solar flares may be due to the spread of the region of turbulent fields once reconnection is initiated at one place. Recent observations demonstrate that gradual flares occur rather in regions with large scale and weak magnetic fields for which Alfvén times are large (Shibata and Magara, 2011). In light of that, the difference in Alfvén times may explain different time scales in gradual and impulsive flares. Further research is necessary for establishing the role of turbulence in changing the time scale of flare evolution.

The LV99 model can find its application in the removal of magnetic flux from the star formation regions. Shu et al. (2006) showed that magnetic field is removed from the star forming core cluster faster than it is allowed by the standard ambipolar diffusion scenario (Tassis and Mouschovias, 2005a,b). Shu et al. (2007) proposed a mechanism using efficient reconnection through "hyper-resistivity". Santos-Lima et al. (2010) performed numerical studies of such a concept, replacing the "hyper-resistivity" with efficient stochastic reconnection. They reported removal of strong anticorrelations of magnetic field through "reconnection diffusion", which can mimic the effect of enhanced Ohmic resistivity.

LV99 showed that fast reconnection of stochastic magnetic field makes the models of strong MHD turbulence selfconsistent, because the critical balance in the GS95 model requires the existence of eddy-type motions perpendicular to 
the magnetic field. In the absence of reconnection this would result in unresolved knots that should drain energy from the cascade. The estimates in LV99 showed that the rates of reconnection predicted by the model are sufficient to resolve magnetic knots within one period.

\subsection{Reasons for slow adaptation of the LV99 model}

The LV99 model of magnetic reconnection in the presence of weakly stochastic magnetic fields was proposed by Lazarian and Vishniac in 1999. However, due to a few objective factors it met with less enthusiasm in the community than, for example, the X-point collisionless reconnection. We believe that there were three major factors responsible for this.

1. The collisionless X-point reconnection was initiated and supported by numerical simulations, while LV99 was a theory. Its numerical testing became possible only recently. The reconnection subject had a history of failed theories and models, which without direct numerical support were taken with a grain of salt.

2. The acceptance of the idea of astrophysical fluids generically being in turbulent state had much less observational support at that time compared to the present day. By now we have much more evidence which allows us to claim that models not taking the pre-existent turbulence has little relevance to astrophysics.

3. The analytical solutions of LV99 were based on the use of GS95 model of turbulence. The GS95 model of turbulence, in fact, was extended LV99 by introducing the concept of local reference frame for turbulent eddies and by extending the GS95 scalings to the sub-Alfvénic case. The GS95 theory was far from being generally accepted at the time of the LV99 publishing.

The situation has changed substantially by now. First of all, GS95 was successfully tested numerically (Cho and Vishniac, 2000; Maron and Goldreich, 2001; Cho et al., 2002) and their ideas have been extended to describing the Alfvénic cascade in compressible MHD turbulence (see Cho and Lazarian, 2002, 2003; Kowal and Lazarian, 2010) ${ }^{3}$. The so-called "Big Power Law in the Sky" indicating the presence of turbulence on scales from tens of parsecs to thousands of kilometres has been extended (Chepurnov and Lazarian, 2010), and the observations of gas and synchrotron emission provided an extended number of direct turbulence

\footnotetext{
${ }^{3}$ There are attempts to modify GS95 theory by supplementing it with additional effects, like dynamical alignment (Boldyrev, 2005, 2006), polarization (Beresnyak and Lazarian, 2006), non-locality Gogoberidze (2007). All these attempts, however, do not change the very nature of the GS95 model. Moreover, some recent studies Beresnyak and Lazarian (2009, 2010); Beresnyak (2011) indicate that the numerical motivation for introducing these attempts may be due to the insufficient inertial range of the simulations involved.
}

measurements confirming their presence (see Burkhart et al., 2010; Gaensler et al., 2011). Finally, the situation has changed with the numerical testing of the LV99 model. The 3-D MHD simulations in Kowal et al. (2009) supported the predictions in the LV99 paper and our present work goes further in testing this model, by including different types of energy injection.

It is worth noting also, that there is some implicit observational evidence in the favour of the LV99 model, like observations of the thick reconnection current outflow regions observed in the Solar flares (Ciaravella and Raymond, 2008). Sych et al. (2009), explaining quasi-periodic pulsations in observed flaring energy releases at an active region above the sunspot, proposed that the wave packets arising from the sunspots can trigger such pulsations. They established a phenomenological relation between oscillations in a sunspot and pulsations in flaring energy releases. This phenomenon can be naturally explained by the LV99 model.

\section{Conclusions}

In this article, we performed additional testing of the LV99 model of fast reconnection under different types of turbulent driving using 3-D numerical simulations. We have introduced a new method of driving turbulence by direct injection of the velocity or magnetic eddies with random locations in the domain. We analysed the dependence of the reconnection speed on the turbulence injection power, on the injection scale, as well as on the viscosity. We found that:

- We observe similar changes of the topology of the magnetic field near the interface of oppositely directed magnetic field lines in models with two different turbulence injection mechanisms. These changes include the fragmentation of the current sheet, favouring multiple simultaneous reconnection events, as well as a substantial increase in the thickness of the outflow of reconnected magnetic flux and matter.

- The relation between the reconnection rate $V_{\text {rec }}$ and turbulent power $P_{\text {inj }}$ remains unchanged under two different mechanisms of energy injection and is confirmed by new models to be $V_{\text {rec }} \sim P_{\text {inj }}^{1 / 2} \sim V_{l}^{2}$, in agreement with the LV99 prediction. Moreover, the injection in magnetic field produces similar effects on the reconnection as injection in velocity, remaining the dependence unaltered.

- The reconnection rate grows with the size of the injected eddies, which can be directly related to the turbulence injection scale. The rate of growth, for the models with old and new driving mechanism, is approximated by $V_{\text {rec }} \sim l_{\text {inj }}^{3 / 4}$ scaling which agrees with the previously obtained scaling. Somewhat steeper LV99 prediction, 
$V_{\text {rec }} \sim l_{\text {inj }}$, could results from limitations in the dynamic range available for study.

- Reconnection in the presence of weak turbulence is only weakly sensitive to viscosity $v$. From performed simulations we obtained a dependence $V_{\text {rec }} \sim v^{-1 / 4}$ for one set of parameters: $P_{\mathrm{inj}}=1.0, k_{\mathrm{inj}}=8, \eta_{u}=10^{-3}$, $B_{0 z}=0.1$.

Acknowledgements. The research of GK is supported by FAPESP grant no. 2009/50053-8, AL is supported by the Center for Magnetic Self-Organization in Laboratory and Astrophysical Plasmas, NSF grant AST-08-08118 and NASA grant NNX09AH78G. The work of ETV is supported by the National Science and Engineering Research Council of Canada. The research of KOM is supported by the Polish Ministry of Science and Higher Education through grants: 92/N-ASTROSIM/2008/0 and 3033/B/H03/2008/35. This research also was supported by the National Science Foundation project TG-AST080005N through TeraGrid resources provided by Texas Advanced Computing Center (TACC: http://www.tacc.utexas.edu). Part of this work was made possible by the facilities of the Shared Hierarchical Academic Research Computing Network (SHARCNET: http://www.sharcnet.ca) and the GALERA supercomputer in the Academic Computer Centre in Gdańsk (TASK:http://www.task.gda.pl).

Edited by: G. Lapenta

Reviewed by: M. Goldstein and another anonymous referee

\section{References}

Alvelius, K.: Random forcing of three-dimensional homogeneous turbulence, Phys. Fl., 11, 1880-1889, 1999.

Ambrosiano, J., Matthaeus, W. H., Goldstein, M. L., and Plante, D.: Test particle acceleration in turbulent reconnecting magnetic fields, J. Geophys. Res., 93, 14383-14400, 1988.

Armstrong, J. W., Rickett, B. J., and Spangler, S. R.: Electron density power spectrum in the local interstellar medium, Astrophys. J., 443, 209-221, 1995.

Balbus, S. A. and Hawley, J. F.: Instability, turbulence, and enhanced transport in accretion disks, Rev. Mod. Phys., 70, 1-53, 1998.

Bastian, T. S., Benz, A. O., and Gary, D. E.: Radio Emission from Solar Flares, Annu. Rev. Astron. Astrophys., 36, 131-188, 1998.

Beck, R.: Magnetic Fields in Spiral Arms and Bars, Astr. Soc. P., 275, 331-342, 2002.

Beresnyak, A.: Spectral Slope and Kolmogorov Constant of MHD Turbulence, Phys. Rev. Lett., 106, 075001, doi:10.1103/PhysRevLett.106.075001, 2011.

Beresnyak, A. and Lazarian, A.: Polarization Intermittency and Its Influence on MHD Turbulence, Astrophys. J. Lett., 640, L175L178, 2006.

Beresnyak, A. and Lazarian, A.: Comparison of Spectral Slopes of Magnetohydrodynamic and Hydrodynamic Turbulence and Measurements of Alignment Effects, Astrophys. J., 702, 1190 $1198,2009$.

Beresnyak, A. and Lazarian, A.: Scaling Laws and Diffuse Locality of Balanced and Imbalanced Magnetohydrodynamic Turbulence, Astrophys. J. Lett., 722, L110-L113, 2010.
Bhattacharjee, A., Ma, Z. W., and Wang, X.: Recent Developments in Collisionless Reconnection Theory: Applications to Laboratory and Astrophysical Plasmas, Lect. Not. Phys., 614, 351-375, 2003.

Bhattacharjee, A., Huang, Y.-M., Yang, H., and Rogers, B.: Fast reconnection in high-Lundquist-number plasmas due to the plasmoid Instability, Phys. Plas., 16, 112102, doi:10.1063/1.3264103, 2009.

Biskamp, D.: Magnetic Reconnection in Plasmas, Astrophys. Space Sci., 242, 165-207, 1996.

Biskamp, D.: Magnetic Reconnection in Plasmas, Cambridge University Press, Cambridge, UK, 387 pp., 2000.

Boldyrev, S.: On the Spectrum of Magnetohydrodynamic Turbulence, Astrophys. J. Lett., 626, L37-L40, 2005.

Boldyrev, S.: $\quad$ Spectrum of Magnetohydrodynamic Turbulence, Phys. Rev. Lett., 96, 115002 , doi:10.1103/PhysRevLett.96.115002, 2006.

Burkhart, B., Stanimirović, S., Lazarian, A., and Kowal, G.: Characterizing Magnetohydrodynamic Turbulence in the Small Magellanic Cloud, Astrophys. J., 708, 1204-1220, 2010.

Cassak, P. A., Shay, M. A., and Drake, J. F.: Catastrophe Model for Fast Magnetic Reconnection Onset, Phys. Rev. Lett., 95, 235002, doi:10.1103/PhysRevLett.95.235002, 2005.

Chepurnov, A. and Lazarian, A.: Extending the Big Power Law in the Sky with Turbulence Spectra from Wisconsin $\mathrm{H} \alpha$ Mapper Data, Astrophys. J., 710, 853-858, 2010.

Chepurnov, A., Lazarian, A., Stanimirović, S., Heiles, C., and Peek, J. E. G.: Velocity Spectrum for H I at High Latitudes, Astrophys. J., 714, 1398-1406, 2010.

Cho, J. and Lazarian, A.: Compressible Sub-Alfvénic MHD Turbulence in Low- $\beta$ Plasmas, Phys. Rev. Lett., 88, 245001, doi:10.1103/PhysRevLett.88.245001, 2002.

Cho, J. and Lazarian, A.: Compressible magnetohydrodynamic turbulence: mode coupling, scaling relations, anisotropy, viscositydamped regime and astrophysical implications, Mon. Not. R. Astron. Soc., 345, 325-339, 2003.

Cho, J., Lazarian, A., and Vishniac, E. T.: Simulations of Magnetohydrodynamic Turbulence in a Strongly Magnetized Medium, Astrophys. J., 564, 291-301, 2002.

Cho, J. and Vishniac, E. T.: The Anisotropy of Magnetohydrodynamic Alfvénic Turbulence, Astrophys. J., 539, 273-282, 2000.

Ciaravella, A. and Raymond, J. C.: The Current Sheet Associated with the 2003 November 4 Coronal Mass Ejection: Density, Temperature, Thickness, and Line Width, Astrophys. J., 686, 1372-1382, 2008.

Crutcher, R. M.: Magnetic Fields in Molecular Clouds: Observations Confront Theory, Astrophys. J., 520, 706-713, 1999.

de Gouveia Dal Pino, E. M. and Lazarian, A.: Constraints on the Acceleration of Ultra-High-Energy Cosmic Rays in Accretioninduced Collapse Pulsars, Astrophys. J., 560, 358-364, 2001.

de Gouveia Dal Pino, E. M. and Lazarian, A.: The role of Violent Magnetic Reconnection on the Production of the Large Scale Superluminal Ejections of the Microquasar GRS 1915+105, arXiv:astro-ph/0307054, 2003.

de Gouveia dal Pino, E. M. and Lazarian, A.: Production of the large scale superluminal ejections of the microquasar GRS $1915+105$ by violent magnetic reconnection, Astron. Astrophys., 441, 845-853, 2005. 
Drake, J. F., Swisdak, M., Che, H., and Shay, M. A.: Electron acceleration from contracting magnetic islands during reconnection, Nature, 443, 553-556, 2006.

Elmegreen, B. G. and Scalo, J.: Interstellar Turbulence I: Observations and Processes, Annu. Rev. Astron. Astrophys., 42, 211273, 2004.

Eyink, G. L., Lazarian, A., and Vishniac, E. T.: Fast Magnetic Reconnection and Spontaneous Stochasticity, Astrophys. J., 743, 51, 2011.

Fitzpatrick, R.: Scaling of forced magnetic reconnection in the Hallmagnetohydrodynamic Taylor problem, Phys. Plas., 11, 937946, 2004

Forbes, T. G.: The nature of Petschek-type reconnection, Earth Planets Space, 53, 423, 2001

Gaensler, B. M., Haverkorn, M., Burkhart, B., Newton-McGee, K. J., Ekers, R. D., Lazarian, A., McClure-Griffiths, N. M., Robishaw, T., Dickey, J. M., and Green, A. J.: Low-Mach-number turbulence in interstellar gas revealed by radio polarization gradients, Nature, 478, 214-217, 2011.

Gogoberidze, G.: On the nature of incompressible magnetohydrodynamic turbulence, Phys. Plas., 14, 022304, doi:10.1063/1.2437753, 2007.

Goldreich, P. and Sridhar, S.: Toward a theory of interstellar turbulence. 2: Strong alfvenic turbulence, Astrophys. J., 438, 763 775, (GS95), 1995.

Goldstein, M. L., Matthaeus, W. H., and Ambrosiano, J. J.: Acceleration of charged particles in magnetic reconnection Solar flares, the magnetosphere, and solar wind, Geophys. Res. Lett., 13, 205-208, 1986.

Gottlieb, S., Ketcheson, D. I. and Shu, C.-W.: High Order Strong Stability Preserving Time Discretizations, J. Sci. Comp., 38, 251-289, 2009.

Hanasz, M., Otmianowska-Mazur, K., Kowal, G., and Lesch, H.: Cosmic-ray-driven dynamo in galactic disks. A parameter study, Astron. Astrophys., 498, 335-346, 2009.

He, Z., Li, X., Fu, D., and Ma, Y.: A 5th order monotonicitypreserving upwind compact difference scheme, Science in China G: Physics and Astronomy, 54, 511-522, 2011.

Hoshino, M.: Stochastic Particle Acceleration in Multiple Magnetic Islands during Reconnection, arXiv:1201.0837, 2012.

Jacobson, A. R. and Moses, R. W.: Nonlocal dc electrical conductivity of a Lorentz plasma in a stochastic magnetic field, Phys. Rev. A, 29, 3335-3342, 1984.

Khanna, R.: Generation of magnetic fields by a gravitomagnetic plasma battery, Mon. Not. R. Astron. Soc., 295, L6-L10, 1998.

Kim, E.-j. and Diamond, P. H.: On Turbulent Reconnection, Astrophys. J., 556, 1052-1065, 2001.

Kotera, K. and Olinto, A. V.: The Astrophysics of Ultrahigh-Energy Cosmic Rays, Annu. Rev. Astron. Astrophys., 49, 119-153, 2011.

Kowal, G., de Gouveia Dal Pino, E. M., and Lazarian, A.: Magnetohydrodynamic Simulations of Reconnection and Particle Acceleration: Three-dimensional Effects, Astrophys. J., 735, 102, 2011.

Kowal, G., de Gouveia Dal Pino, E. M., and Lazarian, A.: Acceleration in Turbulence and Weakly Stochastic Reconnection, Phys. Rev. Lett., in press, 2011.

Kowal, G. and Lazarian, A.: Velocity Field of Compressible Magnetohydrodynamic Turbulence: Wavelet Decomposition and
Mode Scalings, Astrophys. J., 720, 742-756, 2010.

Kowal, G., Lazarian, A., Vishniac, E. T., and Otmianowska-Mazur, K.: Numerical Tests of Fast Reconnection in Weakly Stochastic Magnetic Fields, Astrophys. J., 700, 63-85, 2009.

Kulpa-Dybeł, K., Kowal, G., Otmianowska-Mazur, K., Lazarian, A., and Vishniac, E.: Reconnection in weakly stochastic Bfields in 2-D, Astron. Astrophys., 514, A26, doi:10.1051/00046361/200913218, 2010.

Kulpa-Dybeł, K., Otmianowska-Mazur, K., Kulesza-Żydzik, B., Hanasz, M., Kowal, G., Wóltański, D., and Kowalik, K.: Global Simulations of the Magnetic Field Evolution in Barred Galaxies Under the Influence of the Cosmic-ray-driven Dynamo, Astrophys. J. Lett., 733, L18, doi:10.1088/2041-8205/733/2/L18, 2011.

La Rosa, T. N., Shore, S. N., Joseph, T., Lazio, W., and Kassim, N. E.: The Strength and Structure of the Galactic Center Magnetic Field, J. Phys. Conf. Ser., 54, 10-15, 2006.

Lazarian, A.: Theoretical approaches to particle propagation and acceleration in turbulent intergalactic medium, Astron. Nach. 327, 609, 2006.

Lazarian, A.: Obtaining Spectra of Turbulent Velocity from Observations, Space Sc. Rev., 143, 357-385, 2009.

Lazarian, A. and Pogosyan, D.: Velocity Modification of HI Power Spectrum, Astrophys. J., 537, 720-748, doi:10.1086/309040, 2000.

Lazarian, A. and Vishniac, E. T.: Reconnection in a Weakly Stochastic Field, Astrophys. J., 517, 700-718, (LV99), 1999.

Lazarian, A. and Vishniac, E. T.: Model of Reconnection of Weakly Stochastic Magnetic Field and its Implications, Rev. Mex. Astron. Astrofis. Conf. Ser., 36, 81-88, 2009.

Lazarian, A., Vishniac, E. T., and Cho, J.: Magnetic Field Structure and Stochastic Reconnection in a Partially Ionized Gas, Astrophys. J., 603, 180-197, 2004.

Litvinenko, Y. E.: Particle Acceleration by a Time-Varying Electric Field in Merging Magnetic Fields, Sol. Phys., 216, 189-203, 2003.

Londrillo, P. and Del Zanna, L.: High-Order Upwind Schemes for Multidimensional Magnetohydrodynamics, Astrophys. J., 530, 508-524, 2000.

Loureiro, N. F., Uzdensky, D. A., Schekochihin, A. A., Cowley, S. C., and Yousef, T. A.: Turbulent magnetic reconnection in two dimensions, Mon. Not. R. Astron. Soc., 399, L146-L150, 2009.

Matthaeus, W. H., Ambrosiano, J. J., and Goldstein, M. L.: Particleacceleration by turbulent magnetohydrodynamic reconnection, Phys. Rev. Lett., 53, 1449-1452, 1984.

Matthaeus, W. H. and Lamkin, S. L.: Rapid magnetic reconnection caused by finite amplitude fluctuations, Phys. Fl., 28, 303-307, 1985.

Matthaeus, W. H. and Lamkin, S. L.: Turbulent magnetic reconnection, Phys. Fl., 29, 2513-2534, 1986.

Melrose, D. B.: Acceleration Mechanisms, arXiv:0902.1803, 2009.

Mignone, A.: A simple and accurate Riemann solver for isothermal MHD, J. Comp. Phys., 225, 1427-1441, 2007.

Moffat, H. K.: Magnetic Field Generation in Electrically conducting Fluids, London, UK/New York, NY:Cambridge University Press, 1978.

Maron, J. and Goldreich, P.: Simulations of Incompressible Magnetohydrodynamic Turbulence, Astrophys. J., 554, 1175-1196, 2001. 
Padoan, P., Juvela, M., Kritsuk, A., and Norman, M. L.: The Power Spectrum of Supersonic Turbulence in Perseus, Astrophys. J. Lett., 653, L125-L128, 2006.

Padoan, P., Juvela, M., Kritsuk, A., and Norman, M. L.: The Power Spectrum of Turbulence in NGC 1333: Outflows or Large-Scale Driving?, Astrophys. J. Lett., 707, L153-L157, 2009.

Parker, E. N.: Sweet's Mechanism for Merging Magnetic Fields in Conducting Fluids, J. Geophys. Res., 62, 509-520, 1957.

Parker, E. N.: Fast dynamos, cosmic rays, and the Galactic magnetic field, Astrophys. J., 401, 137-145, 1992.

Petschek, H. E.: Magnetic Field Annihilation, NASA Special Publication, 50, 425-439, 1964.

Pneuman, G. W.: Two-ribbon flares - /Post/-flare loops, Solar Flare Magnetohydrodynamics, 379-428, 1981.

Priest, E. and Forbes, T.: Magnetic Reconnection, Cambridge University Press, Cambridge, UK, 2000.

Santos-Lima, R., Lazarian, A., de Gouveia Dal Pino, E. M., and Cho, J.: Diffusion of Magnetic Field and Removal of Magnetic Flux from Clouds Via Turbulent Reconnection, Astrophys. J., 714, 442-461, 2010.

Schlickeiser, R. and Lerche, I.: Cosmic gas dynamics. I - Basic equations and the dynamics of hot interstellar matter, Astron. Astrophys., 151, 151-156, 1985.

Scholer, M.: Undriven magnetic reconnection in an isolated current sheet, J. Geophys. Res., 94, 8805-8812, 1989.

Servidio, S., Matthaeus, W. H., Shay, M. A., Dmitruk, P., Cassak, P. A., and Wan, M.: Statistics of magnetic reconnection in twodimensional magnetohydrodynamic turbulence, Phys. Plas., 17, 032315, doi:10.1063/1.3368798, 2010.

Shay, M. A., Drake, J. F., Denton, R. E., and Biskamp, D.: Structure of the dissipation region during collisionless magnetic reconnection, J. Geophys. Res., 103, 9165-9176, 1998.

Shay, M. A., Drake, J. F., Swisdak, M., and Rogers, B. N.: The scaling of embedded collisionless reconnection, Phys. Plas., 11, 2199-2213, 2004.

Shibata, K. and Magara, T.: Solar Flares: Magnetohydrodynamic Processes, Liv. Rev. Sol. Phys., 8, http://solarphysics. livingreviews.org/Articles/lrsp-2011-6/, 2011.

Shibata, K. and Tanuma, S.: Plasmoid-induced-reconnection and fractal reconnection, Earth, Planets, and Space, 53, 473-482, 2001.

Shu, F. H., Galli, D., Lizano, S., and Cai, M.: Gravitational Collapse of Magnetized Clouds. II. The Role of Ohmic Dissipation, Astrophys. J., 647, 382-389, 2006.

Shu, F. H., Galli, D., Lizano, S., Glassgold, A. E., and Diamond, P. H.: Mean Field Magnetohydrodynamics of Accretion Disks, Astrophys. J., 665, 535-553, 2007.

Smith, D., Ghosh, S., Dmitruk, P., and Matthaeus, W. H.: Hall and Turbulence Effects on Magnetic Reconnection, Geophys. Res. Lett., 310, L02805, doi:10.1029/2003GL018689, 2004.

Speiser, T. W.: Conductivity without collisions or noise, Plan. Space Sci., 18, 613-1970.

Stanimirović, S. and Lazarian, A.: Velocity and Density Spectra of the Small Magellanic Cloud, Astrophys. J. Lett., 551, L53-L56, 2001.
Suresh, A. and Huynh, H. T.: Accurate Monotonicity-Preserving Schemes with Runge Kutta Time Stepping, J. Comp. Phys., 136, 83-99, 1997.

Sweet, P. A.: The Neutral Point Theory of Solar Flares, Electromagnetic Phenomena in Cosmical Physics, 6, 123-134, , in: Conf. Proc. IAU Symposium 6, Electromagnetic Phenomena in Cosmical Physics, edited by: Lehnert, B., Cambridge, UK, Cambridge University Press, 1958.

Sych, R., Nakariakov, V. M., Karlicky, M., and Anfinogentov, S.: Relationship between wave processes in sunspots and quasiperiodic pulsations in active region flares, Astron. Astrophys., 505, 791-799, 2009.

Tassis, K. and Mouschovias, T. C.: Magnetically Controlled Spasmodic Accretion during Star Formation. I. Formulation of the Problem and Method of Solution, Astrophys. J., 618, 769-782, 2005.

Tassis, K. and Mouschovias, T. C.: Magnetically Controlled Spasmodic Accretion during Star Formation. II. Results, Astrophys. J., 618, 783-794, 2005.

Tóth, G.: The $\nabla \cdot \mathbf{B}=0$ Constraint in Shock-Capturing Magnetohydrodynamics Codes, J. Comp. Phys., 161, 605-652, 2000.

Ugai, M.: Computer studies on development of the fast reconnection mechanism for different resistivity models, Phys. Fl. B, 4, 2953-2963, 1992.

Ugai, M. and Tsuda, T.: Magnetic field-line reconnexion by localized enhancement of resistivity. I - Evolution in a compressible MHD fluid, J. Plas. Phys., 17, 337-356, 1977.

Vallée, J. P.: Observations of the Magnetic Fields Inside and Outside the Milky Way, Starting with Globules ( 1 parsec), Filaments, Clouds, Superbubbles, Spiral Arms, Galaxies, Superclusters, and Ending with the Cosmological Universe's Background Surface (at $\sim 8$ Teraparsecs), Fund. Cosm. Phys., 19, 1-89, 1997.

Vallée, J. P.: Observations of the Magnetic Fields Inside and Outside the Solar System: From Meteorites ( 10 attoparsecs), Asteroids, Planets, Stars, Pulsars, Masers, to Protostellar Cloudlets (i 1 parsec), Fund. Cosm. Phys., 19, 319-422, 1998.

Wang, X., Bhattacharjee, A., and Ma, Z. W.: Scaling of Collisionless Forced Reconnection, Phys. Rev. Lett., 87, 265003, doi:10.1103/PhysRevLett.87.265003, 2001.

Watson, P. G., Oughton, S., and Craig, I. J. D.: The impact of smallscale turbulence on laminar magnetic reconnection, Phys. Plas., 14, 032301, doi:10.1063/1.2458595, 2007.

Yamada, M.: Progress in understanding magnetic reconnection in laboratory and space astrophysical plasmas, Phys. Plas., 14, 058102, doi:10.1063/1.2740595, 2007.

Yan, M., Lee, L. C., and Priest, E. R.: Fast magnetic reconnection with small shock angles, J. Geophys. Res., 97, 8277-8293, 1992.

Zweibel, E. G. and Yamada, M.: Magnetic Reconnection in Astrophysical and Laboratory Plasmas, Annu. Rev. Astron. Astrophys., 47, 291-332, 2009. 